\title{
The Markov additive risk process under an Erlangized dividend barrier strategy
}

\author{
Zhimin Zhang, Eric C.K. Cheung ${ }^{\dagger}$
}

May 26, 2014

\begin{abstract}
In this paper, we consider a Markov additive insurance risk process under a randomized dividend strategy in the spirit of Albrecher et al. (2011). Decisions on whether to pay dividends are only made at a sequence of dividend decision time points whose intervals are $\operatorname{Erlang}(n)$ distributed. At a dividend decision time, if the surplus level is larger than a predetermined dividend barrier, then the excess is paid as a dividend as long as ruin has not occurred. In contrast to Albrecher et al. (2011), it is assumed that the event of ruin is monitored continuously (Avanzi et al. (2013) and Zhang (2014)), i.e. the surplus process is stopped immediately once it drops below zero. The quantities of our interest include the Gerber-Shiu expected discounted penalty function and the expected present value of dividends paid until ruin. Solutions are derived with the use of Markov renewal equations. Numerical examples are given, and the optimal dividend barrier is identified in some cases.
\end{abstract}

Keywords: Markov additive process; Barrier strategy; Inter-dividend-decision times; Gerber-Shiu function; Dividends; Markov renewal equation; Erlangization.

\section{Introduction}

In this paper, we model the surplus of an insurance company via a Markov additive process (MAP) with downward jumps (e.g. Asmussen (2003, Chapter XI)). Let $J=\left\{J_{t}\right\}_{t \geq 0}$ be the underlying environment process, which is a homogeneous irreducible continuous-time Markov chain with finite state space $\mathcal{E}=$ $\{1,2, \ldots, m\}$ and representation $\left(\boldsymbol{\alpha}, \boldsymbol{D}_{0}, \boldsymbol{D}_{1}\right)$. Here $\boldsymbol{\alpha}$ is the initial probability row vector and $\boldsymbol{D}_{0}+\boldsymbol{D}_{1}$ is the intensity matrix. We shall write $\boldsymbol{D}_{0}=\left(D_{0, i j}\right)_{i, j=1}^{m}$ and $\boldsymbol{D}_{1}=\left(D_{1, i j}\right)_{i, j=1}^{m}$. The claim number process $N=\left\{N_{t}\right\}_{t \geq 0}$ of a MAP is controlled by $J$ as follows:

(1) transition of $J$ from state $i$ to state $j$ without any accompanying claim (where $i \neq j$ ) occurs at rate $D_{0, i j} \geq 0$; and

(2) transition of $J$ from state $i$ to state $j$ with an accompanying claim (with the possibility that $i=j$ ) occurs at rate $D_{1, i j} \geq 0$.

Note that for $\boldsymbol{D}_{0}+\boldsymbol{D}_{1}$ to be an intensity matrix, each diagonal element of $\boldsymbol{D}_{0}$ has to be negative and is such that the sum of the elements on each row of $\boldsymbol{D}_{0}+\boldsymbol{D}_{1}$ is zero. The bivariate Markov process $(N, J)$ is called Markovian arrival process. Although in the literature of applied probability, the abbreviation

\footnotetext{
${ }^{*}$ College of Mathematics and Statistics, Chongqing University, Chongqing, 401331, P.R. China.

${ }^{\dagger}$ Department of Statistics and Actuarial Science, The University of Hong Kong, Pokfulam Road, Hong Kong. Corresponding Author: Eric C.K. Cheung (eckc@hku.hk)
} 
'MAP' is also used for Markovian arrival process, we will be using it to refer to the Markov additive process that will be introduced below.

Let $\left\{X_{k}\right\}_{k=1}^{\infty}$ be the sequence of individual claim severities which are positive continuous random variables. It is assumed that the distribution of the claim severity is dependent on the states of the environment process $J$ immediately before and after transition of type (2). More precisely, whenever a transition from $i$ to $j$ is accompanied by a claim, the resulting claim severity has density $f_{i j}$ with mean $\mu_{i j}$. For later use, we let $\boldsymbol{f}(x)=\left(f_{i j}(x)\right)_{i, j=1}^{m}$. In order to account for small fluctuations of the insurer's surplus, we shall use a Brownian motion with zero mean as perturbation. Whenever $J$ is in state $i$, we assume that the insurer collects premium at rate $c_{i}>0$ and the diffusion volatility is $\sigma_{i}>0$. Under these assumptions, the surplus process $U^{\infty}=\left\{U_{t}^{\infty}\right\}_{t \geq 0}$ is defined as

$$
U_{t}^{\infty}=u+\int_{0}^{t} c_{J_{s}} d s-\sum_{k=1}^{N_{t}} X_{k}+\int_{0}^{t} \sigma_{J_{s}} d B_{s}, \quad t \geq 0 .
$$

Here $u \geq 0$ is the initial surplus, and $\left\{B_{t}\right\}_{t \geq 0}$ is a standard Brownian motion starting at zero which is independent of other processes. The process $U^{\infty}$ is a spectrally negative Markov additive process (MAP). For notational convenience, we write $\mathbb{P}_{u, i}\{\cdot\}=\mathbb{P}\left\{\cdot \mid U_{0}^{\infty}=u, J_{0}=i\right\}$ and $\mathbb{E}_{u, i}[\cdot]=\mathbb{E}\left[\cdot \mid U_{0}^{\infty}=u, J_{0}=i\right]$ for $i \in \mathcal{E}$ and $u \geq 0$. The time of ruin of the surplus process $U^{\infty}$ is defined by $\tau^{\infty}=\inf \left\{t>0: U_{t}^{\infty}<0\right\}$ with the convention $\inf \{\emptyset\}=\infty$. The net profit condition is given by

$$
\sum_{i=1}^{m} \pi_{i}\left(c_{i}-\sum_{j=1}^{m} D_{1, i j} \mu_{i j}\right)>0
$$

where $\left(\pi_{1}, \pi_{2}, \ldots, \pi_{m}\right)$ is the stationary probability row vector of $J$. Condition (1.2) ensures that the process (1.1) drifts to infinity in the long run (see e.g. Asmussen (2003, Corollaries 2.7 and 2.9)). Throughout this paper, it is assumed that (1.2) holds.

The class of MAP risk processes (1.1) is known to be very general as it includes the classical compound Poisson risk model (e.g. Asumssen and Albrecher (2010, Section IV)), the Markov-modulated risk process (e.g. Asumssen (1989) and $\mathrm{Lu}$ and Tsai (2007)), the semi-Markovian model by Albrecher and Boxma (2005), and renewal risk process with phase-type inter-arrival times (e.g. Feng (2009a,b)) as special cases. Recently, a lot of contributions have been made to the MAP risk model (with or without diffusion). For example, Cheung and Landriault (2009, Section 4) studied a dividend barrier strategy in which the barrier is allowed to depend on $J$; whereas Zhang et al. (2011) investigated the absolute ruin problem under debit interest. Moreover, Salah and Morales (2012) studied the Gerber-Shiu expected discounted penalty function (Gerber and Shiu (1998)) in a more general spectrally negative MAP risk process; whereas generalizations of the Gerber-Shiu function were analyzed by Cheung and Landriault (2010), Cheung and Feng (2013), and Feng and Shimizu (2014). While the afore-mentioned papers involve analytic derivations of the quantities of interest, we remark that MAP risk processes may also be studied using a more probabilistic approach via connection to Markov-modulated fluid flow (MMFF) processes (e.g. Badescu et al. (2005, 2007), Ahn and Badescu (2007), Ahn et al. (2007)).

In this paper, we shall implement a barrier type dividend strategy in the MAP risk process described above. Recall that in the traditional dividend barrier strategy, the insurer pays dividends to its shareholders immediately whenever the surplus process reaches a fixed barrier level if ruin has not yet occurred (e.g. Gerber (1979), Lin et al. (2003) and Gerber and Shiu (2004)). However, when the surplus process contains a diffusion component, dividend payments may occur many times in a small time interval due 
to the existence of small fluctuations. Following the ideas as in Albrecher et al. (2011), one way to get around this problem is to assume that decisions are only made at discrete time points on whether lump sum dividend payments are paid. More specifically, we let $\left\{Z_{i}\right\}_{i=1}^{\infty}$ be the sequence of dividend decision times. At time $Z_{i}$, if the surplus level $x$ is larger than a given barrier $b>0$, then a lump sum dividend payment of size $x-b$ is paid to the shareholders of the insurance company. To give the mathematical descriptions of the modified surplus process $U^{b}=\left\{U_{t}^{b}\right\}_{t \geq 0}$ with dividends, the auxiliary process $U_{i}^{*}=\left\{U_{i}^{*}(t)\right\}_{t \geq 0}$ is introduced for $i=1,2, \ldots$. The dynamics of $U^{b}$ and $U_{i}^{*}$ can be jointly described recursively via

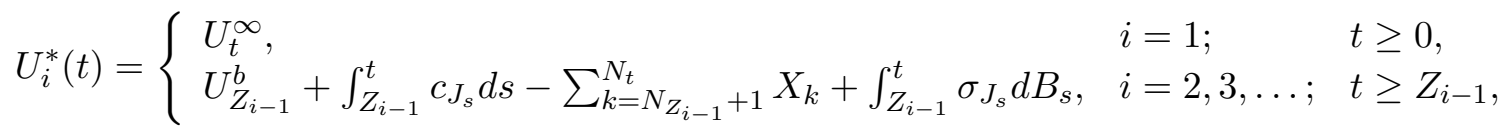

and for $i=1,2, \ldots$,

$$
U_{t}^{b}= \begin{cases}U_{i}^{*}(t), & Z_{i-1}<t<Z_{i} \\ \min \left(U_{i}^{*}\left(Z_{i}\right), b\right), & t=Z_{i}\end{cases}
$$

Without loss of generality, it is assumed that $Z_{0}=0$ - in the above definition, and therefore $U_{0}^{b}=u$ even if $U_{0}^{\infty}=u>b$. This means that time 0 is not assumed to be a dividend decision time. Unlike Albrecher et al. (2011, 2013) who assumed that the event of ruin is only checked at the times $\left\{Z_{i}\right\}_{i=1}^{\infty}$, Zhang (2014) studied a variant of the model where solvency is monitored continuously as in the traditional case (see also Avanzi et al. (2013) for the corresponding variant in a dual risk model). We shall adopt the traditional definition of ruin in the sense that the surplus process is stopped immediately once it drops below zero. Hence, the time of ruin of $U^{b}$ is defined by $\tau^{b}=\inf \left\{t>0: U_{t}^{b}<0\right\}$. Let $T_{1}=Z_{1}$ be the first dividend decision time, and $T_{i}=Z_{i}-Z_{i-1}$ be the $i$ th inter-dividend-decision time (i.e. the interval between the $(i-1)$ th and the $i$ th dividend decision times) for $i=2,3, \ldots$. For the rest of the paper, it is assumed that $\left\{T_{i}\right\}_{i=1}^{\infty}$ forms a sequence of independent and identically distributed random variables distributed as $T$ with the $\operatorname{Erlang}(n)$ density

$$
f_{T}(t)=\frac{\beta^{n} t^{n-1} e^{-\beta t}}{(n-1) !}, \quad t>0 .
$$

Here $n$ is the shape parameter which is a positive integer, and $\beta>0$ is the scale parameter. It is assumed that $\left\{T_{i}\right\}_{i=1}^{\infty}$ is independent of all the attributes of the barrier-free process $U^{\infty}$. The choice of the $\operatorname{Erlang}(n)$ distribution is motivated by the Erlangization techniques proposed by Asmussen et al. (2002) in solving finite-time ruin problems (see also e.g. Stanford et al. (2005, 2011) and Ramaswami et al. (2008)). Indeed, if we fix the mean $\mathbb{E}[T]=n / \beta=h$ and increase $n$ (and $\beta$ as well), then $T$ converges in distribution to a point mass at $h$. Hence, one can approximate the situation where the inter-dividend-decision times are deterministic.

The Gerber-Shiu expected discounted penalty function, or Gerber-Shiu function in short, has been analyzed extensively in increasingly complex risk models since its introduction by Gerber and Shiu (1998). It unifies the study of various ruin-related quantities such as the time of ruin and the deficit at ruin. In this paper, we are interested in the Gerber-Shiu function pertaining to $U^{b}$ defined as (given initial state $i \in \mathcal{E}$ and initial surplus $u \geq 0$ )

$$
\phi_{i}(u ; b)=\mathbb{E}_{u, i}\left[e^{-\delta \tau^{b}} w\left(\left|U_{\tau^{b}}^{b}\right|\right)\right] .
$$

Here $\delta \geq 0$ can be interpreted as the force of interest or the Laplace transform argument with respect to $\tau^{b}$, and $w:[0, \infty) \rightarrow[0, \infty)$ is the so-called penalty function that depends on the deficit at ruin $\left|U_{\tau^{b}}^{b}\right|$. It is assumed that $w$ satisfies some mild integrability conditions. Note that the indicator of the event 
$\left\{\tau^{b}<\infty\right\}$ is not necessary in the definition (1.3), since ruin occurs almost surely as the surplus can never exceed level $b$ at the dividend decision times. Because of the perturbation, ruin may occur due to a claim or by diffusion. Thus, one may rewrite (1.3) as (e.g. Gerber and Landry (1998) and Tsai and Willmot (2002))

$$
\phi_{i}(u ; b)=w(0) \mathbb{E}_{u, i}\left[e^{-\delta \tau^{b}} \mathbf{1}_{\left\{U_{\tau^{b}}^{b}=0\right\}}\right]+\mathbb{E}_{u, i}\left[e^{-\delta \tau^{b}} w\left(\left|U_{\tau^{b}}^{b}\right|\right) \mathbf{1}_{\left\{U_{\tau^{b}}^{b}<0\right\}}\right],
$$

where $\mathbf{1}_{A}$ stands for the indicator function of the event $A$. It is clear from (1.4) that if we are only interested in the contribution by ruin due to diffusion, one can simply let $w(y)=0$ for $y>0$. In contrast, the case where ruin is caused by a claim can be retrieved by letting $w(0)=0$. For later use, we also let $\phi_{i}(u ; \infty)$ be the Gerber-Shiu function associated with the barrier-free model $U^{\infty}$. Another quantity of interest in this paper is the expected discounted dividends paid until ruin defined by (for a force of interest of $\delta>0$ )

$$
V_{i}(u ; b)=\mathbb{E}_{u, i}\left[\sum_{i=1}^{\infty} e^{-\delta Z_{i}}\left(U_{Z_{i}-}^{b}-b\right)_{+} \mathbf{1}_{\left\{Z_{i}<\tau^{b}\right\}}\right],
$$

where $a_{+}=\max (a, 0)$. In the corporate finance literature, the expectation of the present value of dividends represents the value of the firm. Therefore, under the current barrier type dividend strategy, the shareholders' interest would be to find the optimal barrier $b^{*}$ that maximizes $V_{i}(u ; b)$ with respect to $b$.

The remainder of this paper is structured as follows. In Section 2, some preliminary results and notations that will be used throughout are presented. Expressions for the Gerber-Shiu function (1.3) and the expected discounted dividends (1.5) are derived in Sections 3 and 4 respectively using Markov renewal equations. Examples along with numerical illustrations are then given in Section 5. The Appendix is concerned with the proofs of the continuity and smooth pasting conditions required in the derivations.

\section{Preliminaries}

In this paper, matrix notations will be used extensively. We shall use $\boldsymbol{O}$ to denote the zero matrix or vector with appropriate dimension known from the context. For a positive integer $k$, let $\boldsymbol{E}_{k}$ be the identity matrix of dimension $k$, and $\boldsymbol{e}_{k}$ be a column vector of ones with length $k$. For two arbitrary square matrices $\boldsymbol{A}=\left(a_{i j}\right)_{i, j=1}^{k}$ and $\boldsymbol{B}=\left(b_{i j}\right)_{i, j=1}^{k}$, the Hadamard product (i.e. entrywise multiplication) is defined as $\boldsymbol{A} \circ \boldsymbol{B}=\left(a_{i j} b_{i j}\right)_{i, j=1}^{k}$. The notation $\boldsymbol{A}^{\top}$ denotes the transpose of a matrix or vector $\boldsymbol{A}$. In addition, we denote the Laplace transform of a function defined on $[0, \infty)$ (which is not necessarily a probability density) by adding a hat on it. For example, for $\Re(s) \geq 0$, one has $\widehat{f}_{i j}(s)=\int_{0}^{\infty} e^{-s x} f_{i j}(x) d x$. Any integral with respect to a matrix-valued function is taken element-wise. For example, $\widehat{\boldsymbol{f}}(s)=\int_{0}^{\infty} e^{-s x} \boldsymbol{f}(x) d x=$ $\left(\widehat{f}_{i j}(s)\right)_{i, j=1}^{m}$.

The notion of the matrix Dickson-Hipp operator plays an important part in our analysis. The matrix version of the Dickson-Hipp operator was first introduced by Feng (2009b) as an extension of the classical scalar counterpart proposed by Dickson and Hipp (2001). For a square matrix $\boldsymbol{A}$ having eigenvalues on the right-half of the complex plane, the matrix Dickson-Hipp operator $\mathcal{T}_{\boldsymbol{A}}$ is defined as

$$
\mathcal{T}_{\boldsymbol{A}} \boldsymbol{h}(x)=\int_{x}^{\infty} e^{-\boldsymbol{A}(y-x)} \boldsymbol{h}(y) d y=\int_{0}^{\infty} e^{-\boldsymbol{A} y} \boldsymbol{h}(x+y) d y, \quad x \geq 0,
$$

where $\boldsymbol{h}$ is a matrix-valued function with appropriate dimension (such that the multiplication $\boldsymbol{A} \boldsymbol{h}$ makes sense) satisfying some integrability conditions (such that the above integral exists). When $\boldsymbol{A}$ reduces 
to a scalar $r$ with non-negative real part, then $\mathcal{T}_{r}$ is the classical Dickson-Hipp operator. If $x=0$ and $\boldsymbol{A}=s \boldsymbol{E}_{k}$ for $\Re(s) \geq 0$ and some positive integer $k$ such that $\boldsymbol{E}_{k} \boldsymbol{h}$ makes sense, then (2.1) is equivalent to the Laplace transform of $\boldsymbol{h}$ with argument $s$, namely $\widehat{\boldsymbol{h}}(s)$. An appealing property of the (matrix) Dickson-Hipp operator is the commutative property. In particular, if the square matrices $\boldsymbol{A}_{1}$ and $\boldsymbol{A}_{2}$ commute (i.e. $\boldsymbol{A}_{1} \boldsymbol{A}_{2}=\boldsymbol{A}_{2} \boldsymbol{A}_{1}$ ) and $\boldsymbol{A}_{1}-\boldsymbol{A}_{2}$ is nonsingular, then Feng (2009b, Lemma 2.1) showed that

$$
\mathcal{T}_{\boldsymbol{A}_{1}} \mathcal{T}_{\boldsymbol{A}_{2}} \boldsymbol{h}(x)=\mathcal{T}_{\boldsymbol{A}_{2}} \mathcal{T}_{\boldsymbol{A}_{1}} \boldsymbol{h}(x)=\left(\boldsymbol{A}_{1}-\boldsymbol{A}_{2}\right)^{-1}\left(\mathcal{T}_{\boldsymbol{A}_{2}} \boldsymbol{h}(x)-\mathcal{T}_{\boldsymbol{A}_{1}} \boldsymbol{h}(x)\right), \quad x \geq 0 .
$$

Whenever a function under consideration has two arguments $u$ and $b$, any derivative, Laplace transform or Dickson-Hipp operator is assumed to be taken with respect to the first argument $u$ by default.

Next, we introduce some preliminaries on MAP. Let $\boldsymbol{\Delta}_{\sigma^{2}}=\operatorname{diag}\left(\sigma_{1}^{2}, \ldots, \sigma_{m}^{2}\right)$ and $\boldsymbol{\Delta}_{c}=\operatorname{diag}\left(c_{1}, \ldots, c_{m}\right)$. From Asmussen (2003, Proposition XI.2.2), the matrix cumulant generating function of $U^{\infty}$ is given by

$$
\boldsymbol{G}(s)=\frac{1}{2} s^{2} \boldsymbol{\Delta}_{\sigma^{2}}+s \boldsymbol{\Delta}_{c}+\boldsymbol{D}_{0}+\boldsymbol{D}_{1} \circ \widehat{\boldsymbol{f}}(s)
$$

for $s \in \mathbb{C}$ such that the integral in the last term exists. Note that $\boldsymbol{G}(s)$ is well defined at least for $\Re(s) \geq 0$. There exists a matrix $\boldsymbol{Q}_{\gamma}$ that satisfies, for a given $\gamma \geq 0$,

$$
\frac{1}{2} \boldsymbol{Q}_{\gamma}^{2} \boldsymbol{\Delta}_{\sigma^{2}}+\boldsymbol{Q}_{\gamma} \boldsymbol{\Delta}_{c}-\gamma \boldsymbol{E}_{m}+\boldsymbol{D}_{0}+\int_{0}^{\infty} e^{-\boldsymbol{Q}_{\gamma} x}\left(\boldsymbol{D}_{1} \circ \boldsymbol{f}(x)\right) d x=\boldsymbol{O} .
$$

The existence of $\boldsymbol{Q}_{\gamma}$ is known from Breuer (2008, Theorems 1 and 2) and Feng and Shimizu (2014, Lemma 3.2 ) whereas the relation of $\boldsymbol{Q}_{\gamma}$ to the intensity matrix of the time-reversed version of the MAP risk model has been discussed by e.g. Zhang et al. (2011, Section 3) and Salah and Morales (2012, Section 4). In particular, the matrix $\boldsymbol{Q}_{\gamma}$ can be computed using either an iterative approach (Breuer (2008, Theorem 2)) or the more well-known eigenvalue/eigenvector method (e.g. Zhang et al. (2011, Lemma 1), and Cheung and Feng (2013, Appendix)). We shall describe the latter method which is indeed linked to the more classical form of the Lundberg's equation (in $\xi$ ), namely

$$
\operatorname{det}\left(\boldsymbol{G}(\xi)-\gamma \boldsymbol{E}_{m}\right)=0 .
$$

It follows from Feng and Shimizu (2014, Lemma 3.2) that the above equation has exactly $m$ roots with non-negative real parts. These roots are denoted by $\rho_{\gamma, 1}, \ldots, \rho_{\gamma, m}$. Throughout this paper, we suppose that $\boldsymbol{Q}_{\gamma}$ is diagonalizable. A sufficient condition for $\boldsymbol{Q}_{\gamma}$ to be diagonalizable is that $\rho_{\gamma, 1}, \ldots, \rho_{\gamma, m}$ are distinct. Then $\boldsymbol{Q}_{\gamma}$ admits the representation $\boldsymbol{Q}_{\gamma}=\boldsymbol{B}_{\gamma}^{-1} \boldsymbol{\Delta}_{\rho_{\gamma}} \boldsymbol{B}_{\gamma}$ (see Zhang et al. (2011, Lemma 1)). Here $\boldsymbol{\Delta}_{\rho_{\gamma}}=\operatorname{diag}\left(\rho_{\gamma, 1}, \ldots, \rho_{\gamma, m}\right)$ is the matrix of eigenvalues and $\boldsymbol{B}_{\gamma}=\left(\boldsymbol{b}_{\gamma, 1}^{\top}, \ldots, \boldsymbol{b}_{\gamma, m}^{\top}\right)^{\top}$ is the matrix containing the corresponding eigenvectors. In particular, for each fixed $i=1,2, \ldots, m$, the left eigenvector $\boldsymbol{b}_{\gamma, i}$ is a non-trivial solution of the equation (in $\boldsymbol{b}$ )

$$
\boldsymbol{b}\left[\boldsymbol{G}\left(\rho_{\gamma, i}\right)-\gamma \boldsymbol{E}_{m}\right]=\boldsymbol{O} .
$$

It is instructive to note that since the situations in which there are multiple roots to the Lundberg's equation (2.4) are rare, the diagonalizability assumption imposed on $\boldsymbol{Q}_{\gamma}$ is not restrictive. Interested readers are referred to Ji and Zhang (2012) for the treatment of risk models with multiple Lundberg's roots.

\section{The Gerber-Shiu function}

This section aims at deriving the solution to the Gerber-Shiu function. Note that the Erlang $(n)$ interdividend-decision time $T$ can be regarded as the sum of $n$ independent and identically distributed exponential variables. To ease our analysis, for $k=1,2, \ldots, n$ we define $\phi_{k, i}(u ; b)$ to be the Gerber-Shiu 
function under the same conditions as $\phi_{i}(u ; b)$, except that the time until the first (not between all) dividend decision time is $\operatorname{Erlang}(n-k+1)$ distributed. Obviously, one has that $\phi_{i}(u ; b)=\phi_{1, i}(u ; b)$. The introduction of these auxiliary functions will enable us to capture the underlying phase-type structure of the problem.

\subsection{System of integro-differential equations}

We can start by considering the competition between the state transition of $J$ and the phase transition of the first dividend decision time over a very small time interval $[0, h]$ for $i \in \mathcal{E}$ and $u>0$. For $k=1,2, \ldots, n-1$, no dividends will be payable within the interval and one has

$$
\begin{aligned}
\phi_{k, i}(u ; b)= & \left(1-\left(-D_{0, i i}+\beta\right) h\right) e^{-\delta h} \mathbb{E}\left[\phi_{k, i}\left(u+c_{i} h+\sigma_{i} B_{h} ; b\right)\right]+\sum_{j=1, j \neq i}^{m} D_{0, i j} h e^{-\delta h} \mathbb{E}\left[\phi_{k, j}\left(u+c_{i} h+\sigma_{i} B_{h} ; b\right)\right] \\
& +\sum_{j=1}^{m} D_{1, i j} h e^{-\delta h} \mathbb{E}\left[\gamma_{k, i j}\left(u+c_{i} h+\sigma_{i} B_{h} ; b\right)+\omega_{i j}\left(u+c_{i} h+\sigma_{i} B_{h}\right)\right] \\
& +\beta h e^{-\delta h} \mathbb{E}\left[\phi_{k+1, i}\left(u+c_{i} h+\sigma_{i} B_{h} ; b\right)\right]+o(h)
\end{aligned}
$$

where $\gamma_{k, i j}(u ; b)=\int_{0}^{u} \phi_{k, j}(u-x ; b) f_{i j}(x) d x$ and $\omega_{i j}(u)=\int_{u}^{\infty} w(x-u) f_{i j}(x) d x$. Applying Taylor's expansion to (3.1), dividing by $h$, letting $h \rightarrow 0$ and rearranging terms, we obtain

$$
\begin{aligned}
0= & \frac{\sigma_{i}^{2}}{2} \phi_{k, i}^{\prime \prime}(u ; b)+c_{i} \phi_{k, i}^{\prime}(u ; b)-(\delta+\beta) \phi_{k, i}(u ; b)+\sum_{j=1}^{m} D_{0, i j} \phi_{k, j}(u ; b)+\sum_{j=1}^{m} D_{1, i j}\left(\gamma_{k, i j}(u ; b)+\omega_{i j}(u)\right) \\
& +\beta \phi_{k+1, i}(u ; b), \quad k=1,2, \ldots, n-1 .
\end{aligned}
$$

For $k=n$, the analysis is essentially the same, except that dividends will be paid if the surplus is above $b$ when the first dividend decision time occurs. This leads us to

$$
\begin{aligned}
0= & \frac{\sigma_{i}^{2}}{2} \phi_{n, i}^{\prime \prime}(u ; b)+c_{i} \phi_{n, i}^{\prime}(u ; b)-(\delta+\beta) \phi_{n, i}(u ; b)+\sum_{j=1}^{m} D_{0, i j} \phi_{n, j}(u ; b)+\sum_{j=1}^{m} D_{1, i j}\left(\gamma_{n, i j}(u ; b)+\omega_{i j}(u)\right) \\
& +\beta\left(\phi_{1, i}(u ; b) \mathbf{1}_{\{0<u \leq b\}}+\phi_{1, i}(b ; b) \mathbf{1}_{\{u>b\}}\right) .
\end{aligned}
$$

Define $\phi_{k}(u ; b)=\left(\phi_{k, 1}(u ; b), \ldots, \phi_{k, m}(u ; b)\right)^{\top}$ for $k=1,2, \ldots, n$. The integro-differential equations (3.2) and (3.3) can then be rewritten in matrix form as

$$
\begin{aligned}
\boldsymbol{O}= & \left(\frac{1}{2} \boldsymbol{\Delta}_{\sigma^{2}} \frac{d^{2}}{d u^{2}}+\boldsymbol{\Delta}_{c} \frac{d}{d u}-(\delta+\beta) \boldsymbol{E}_{m}+\boldsymbol{D}_{0}\right) \boldsymbol{\phi}_{k}(u ; b)+\int_{0}^{u}\left(\boldsymbol{D}_{1} \circ \boldsymbol{f}(x)\right) \boldsymbol{\phi}_{k}(u-x ; b) d x+\beta \boldsymbol{\phi}_{k+1}(u ; b) \\
& +\boldsymbol{\zeta}(u), \quad k=1,2, \ldots, n-1
\end{aligned}
$$

and

$$
\begin{aligned}
\boldsymbol{O}= & \left(\frac{1}{2} \boldsymbol{\Delta}_{\sigma^{2}} \frac{d^{2}}{d u^{2}}+\boldsymbol{\Delta}_{c} \frac{d}{d u}-(\delta+\beta) \boldsymbol{E}_{m}+\boldsymbol{D}_{0}\right) \boldsymbol{\phi}_{n}(u ; b)+\int_{0}^{u}\left(\boldsymbol{D}_{1} \circ \boldsymbol{f}(x)\right) \boldsymbol{\phi}_{n}(u-x ; b) d x+\beta \boldsymbol{\phi}_{1}(u ; b) \mathbf{1}_{\{0<u \leq b\}} \\
& +\beta \boldsymbol{\phi}_{1}(b ; b) \mathbf{1}_{\{u>b\}}+\boldsymbol{\zeta}(u),
\end{aligned}
$$

where $\boldsymbol{\zeta}(u)=\left(\boldsymbol{D}_{1} \circ \boldsymbol{\omega}(u)\right) \boldsymbol{e}_{m}$ and $\boldsymbol{\omega}(u)=\left(\omega_{i j}(u)\right)_{i, j=1}^{m}$.

A trivial boundary condition for the system comprising (3.4) and (3.5) is given by

$$
\phi_{k}(0 ; b)=w(0) \boldsymbol{e}_{m}, \quad k=1,2, \ldots, n,
$$


since ruin occurs immediately with zero initial surplus. In addition, we assert that the continuity condition

$$
\phi_{k}(b-; b)=\phi_{k}(b+; b), \quad k=1,2, \ldots, n,
$$

and the smooth pasting condition

$$
\phi_{k}^{\prime}(b-; b)=\phi_{k}^{\prime}(b+; b), \quad k=1,2, \ldots, n,
$$

hold at the barrier $b$. See Appendix for further discussions of (3.7) and (3.8).

\subsection{The case $0<u<b$}

In this subsection, we will solve (3.4) and (3.5) when $0<u<b$ apart from some unknown constants. Using the notion of Kronecker product, we define the square matrices $\tilde{\boldsymbol{\Delta}}_{\sigma^{2}}=\boldsymbol{E}_{n} \otimes \boldsymbol{\Delta}_{\sigma^{2}}, \tilde{\boldsymbol{\Delta}}_{c}=\boldsymbol{E}_{n} \otimes \boldsymbol{\Delta}_{c}$, $\tilde{\boldsymbol{D}}_{1}=\boldsymbol{E}_{n} \otimes \boldsymbol{D}_{1}, \tilde{\boldsymbol{f}}(x)=\boldsymbol{E}_{n} \otimes \boldsymbol{f}(x)$, and

$$
\tilde{\boldsymbol{D}}_{0}=\left(\begin{array}{ccccc}
\boldsymbol{D}_{0}-\beta \boldsymbol{E}_{m} & \beta \boldsymbol{E}_{m} & \boldsymbol{O} & \cdots & \boldsymbol{O} \\
\boldsymbol{O} & \boldsymbol{D}_{0}-\beta \boldsymbol{E}_{m} & \beta \boldsymbol{E}_{m} & \cdots & \boldsymbol{O} \\
\vdots & \vdots & \ddots & \ddots & \vdots \\
\boldsymbol{O} & \boldsymbol{O} & \boldsymbol{O} & \cdots & \beta \boldsymbol{E} \\
\beta \boldsymbol{E}_{m} & \boldsymbol{O} & \boldsymbol{O} & \cdots & \boldsymbol{D}_{0}-\beta \boldsymbol{E}_{m}
\end{array}\right)
$$

all of dimension $m n$. (The above matrix is understood to be $\boldsymbol{D}_{0}$ when $n=1$.) Further define the column vectors $\underline{\phi}(u ; b)=\left(\phi_{1}^{\top}(u ; b), \ldots, \phi_{n}^{\top}(u ; b)\right)^{\top}$ and $\underline{\boldsymbol{\zeta}}(u)=\boldsymbol{e}_{n} \otimes \boldsymbol{\zeta}(u)$. Then (3.4) and (3.5) can be neatly combined to yield

$$
\left(\frac{1}{2} \tilde{\boldsymbol{\Delta}}_{\sigma^{2}} \frac{d^{2}}{d u^{2}}+\tilde{\boldsymbol{\Delta}}_{c} \frac{d}{d u}-\delta \boldsymbol{E}_{m n}+\tilde{\boldsymbol{D}}_{0}\right) \underline{\boldsymbol{\phi}}(u ; b)+\int_{0}^{u}\left(\tilde{\boldsymbol{D}}_{1} \circ \tilde{\boldsymbol{f}}(x)\right) \underline{\boldsymbol{\phi}}(u-x ; b) d x+\underline{\boldsymbol{\zeta}}(u)=\boldsymbol{O}, \quad 0<u<b,
$$

which is a non-homogeneous matrix integro-differential equation. From the theory of integro-differential equations, the general solution of (3.9) can be expressed in terms of one of its particular solution plus a fundamental set of solutions of the homogeneous system. Hence we first identify a particular solution as follows. Define $\phi(u ; \infty)=\left(\phi_{1}(u ; \infty), \ldots, \phi_{m}(u ; \infty)\right)^{\top}$ for the barrier-free model $U^{\infty}$. Note that the integro-differential equation satisfied by $\phi(u ; \infty)$ can be obtained from (3.5) by setting $n=1$ and taking the limit $b \rightarrow \infty$. Thus, we have

$$
\left(\frac{1}{2} \boldsymbol{\Delta}_{\sigma^{2}} \frac{d^{2}}{d u^{2}}+\boldsymbol{\Delta}_{c} \frac{d}{d u}-\delta \boldsymbol{E}_{m}+\boldsymbol{D}_{0}\right) \boldsymbol{\phi}(u ; \infty)+\int_{0}^{u}\left(\boldsymbol{D}_{1} \circ \boldsymbol{f}(x)\right) \boldsymbol{\phi}(u-x ; \infty) d x+\boldsymbol{\zeta}(u)=\boldsymbol{O}, \quad u>0,
$$

from which one can easily deduce that $\underline{\phi}(u ; \infty)=\boldsymbol{e}_{n} \otimes \phi(u ; \infty)$ is a particular solution of (3.9), i.e.

$$
\left(\frac{1}{2} \tilde{\boldsymbol{\Delta}}_{\sigma^{2}} \frac{d^{2}}{d u^{2}}+\tilde{\boldsymbol{\Delta}}_{c} \frac{d}{d u}-\delta \boldsymbol{E}_{m n}+\tilde{\boldsymbol{D}}_{0}\right) \underline{\boldsymbol{\phi}}(u ; \infty)+\int_{0}^{u}\left(\tilde{\boldsymbol{D}}_{1} \circ \tilde{\boldsymbol{f}}(x)\right) \underline{\phi}(u-x ; \infty) d x+\underline{\boldsymbol{\zeta}}(u)=\boldsymbol{O}, \quad u>0 .
$$

Next, let $\underline{\boldsymbol{v}}_{\delta}(u)$ be a vector valued function of length $m n$ such that $\underline{\boldsymbol{v}}_{\delta}(0)=\boldsymbol{O}$ and it satisfies the homogeneous version of (3.9), namely

$$
\left(\frac{1}{2} \tilde{\boldsymbol{\Delta}}_{\sigma^{2}} \frac{d^{2}}{d u^{2}}+\tilde{\boldsymbol{\Delta}}_{c} \frac{d}{d u}-\delta \boldsymbol{E}_{m n}+\tilde{\boldsymbol{D}}_{0}\right) \underline{\boldsymbol{v}}_{\delta}(u)+\int_{0}^{u}\left(\tilde{\boldsymbol{D}}_{1} \circ \tilde{\boldsymbol{f}}(x)\right) \underline{\boldsymbol{v}}_{\delta}(u-x) d x=\boldsymbol{O}, \quad u>0 .
$$


By taking Laplace transforms on both sides of (3.11), we obtain

$$
\left(\frac{1}{2} s^{2} \tilde{\boldsymbol{\Delta}}_{\sigma^{2}}+s \tilde{\boldsymbol{\Delta}}_{c}-\delta \boldsymbol{E}_{m n}+\tilde{\boldsymbol{D}}_{0}+\tilde{\boldsymbol{D}}_{1} \circ \widehat{\tilde{\boldsymbol{f}}}(s)\right) \underline{\widehat{\underline{v}}}_{\delta}(s)=\frac{1}{2} \tilde{\boldsymbol{\Delta}}_{\sigma^{2}} \underline{\boldsymbol{v}}_{\delta}^{\prime}(0),
$$

leading to

$$
\underline{\boldsymbol{v}}_{\delta}(u)=\underline{\boldsymbol{L}}_{\delta}(u)\left(\frac{1}{2} \tilde{\boldsymbol{\Delta}}_{\sigma^{2}} \underline{\boldsymbol{v}}_{\delta}^{\prime}(0)\right), \quad u \geq 0
$$

where

$$
\underline{\boldsymbol{L}}_{\delta}(u)=\left(\begin{array}{ccc}
\boldsymbol{L}_{\delta, 1,1}(u) & \cdots & \boldsymbol{L}_{\delta, 1, n}(u) \\
\vdots & \ddots & \vdots \\
\boldsymbol{L}_{\delta, n, 1}(u) & \cdots & \boldsymbol{L}_{\delta, n, n}(u)
\end{array}\right)=\mathcal{L}^{-1}\left(\left(\frac{1}{2} s^{2} \tilde{\boldsymbol{\Delta}}_{\sigma^{2}}+s \tilde{\boldsymbol{\Delta}}_{c}-\delta \boldsymbol{E}_{m n}+\tilde{\boldsymbol{D}}_{0}+\tilde{\boldsymbol{D}}_{1} \circ \widehat{\tilde{\boldsymbol{f}}}(s)\right)^{-1}\right) .
$$

Here each sub-matrix $\boldsymbol{L}_{\delta, i, j}(u)$ is a square matrix of dimension $m$, and $\mathcal{L}^{-1}$ represents the inverse Laplace transform operator.

Now, by taking the difference of (3.9) and (3.10), we note that $\underline{\phi}(u ; b)-\underline{\phi}(u ; \infty)$ satisfies $(3.11)$ for $0<$ $u<b$. Moreover, using $\underline{\phi}(0 ; \infty)=w(0) \boldsymbol{e}_{m n}$ and $(3.6)$, it is clear that the condition $\underline{\phi}(0 ; b)-\underline{\phi}(0 ; \infty)=\boldsymbol{O}$ holds true. Thus, (3.12) implies that we must have

$$
\underline{\phi}(u ; b)=\underline{\phi}(u ; \infty)+\underline{\boldsymbol{L}}_{\delta}(u)\left(\boldsymbol{k}_{1}^{\top}, \ldots, \boldsymbol{k}_{n}^{\top}\right)^{\top}, \quad 0 \leq u \leq b,
$$

where $\boldsymbol{k}_{1}, \ldots, \boldsymbol{k}_{n}$ are unknown column vectors of constants, each of length $m$, that are to be determined later (as in (3.48)). It remains to derive exact expressions for $\underline{\phi}(u ; \infty)$ and $\underline{\boldsymbol{L}}_{\delta}(u)$. The derivation relies on the fact that

$$
\frac{1}{2} s^{2} \tilde{\boldsymbol{\Delta}}_{\sigma^{2}}+s \tilde{\boldsymbol{\Delta}}_{c}+\tilde{\boldsymbol{D}}_{0}+\tilde{\boldsymbol{D}}_{1} \circ \widehat{\tilde{\boldsymbol{f}}}(s)
$$

is the matrix cumulant generating function of a certain MAP with intensity matrix $\tilde{\boldsymbol{D}}_{0}+\tilde{\boldsymbol{D}}_{1}$. Hence, it follows from Section 2 (see (2.3)) that there exists a matrix $\tilde{\boldsymbol{Q}}_{\delta}$ (assumed to be diagonalizable) such that

$$
\frac{1}{2} \tilde{\boldsymbol{Q}}_{\delta}^{2} \tilde{\boldsymbol{\Delta}}_{\sigma^{2}}+\tilde{\boldsymbol{Q}}_{\delta} \tilde{\boldsymbol{\Delta}}_{c}-\delta \boldsymbol{E}_{m n}+\tilde{\boldsymbol{D}}_{0}+\int_{0}^{\infty} e^{-\tilde{\boldsymbol{Q}}_{\delta} x}\left(\tilde{\boldsymbol{D}}_{1} \circ \tilde{\boldsymbol{f}}(x)\right) d x=\boldsymbol{O},
$$

and the eigenvalues of $\tilde{\boldsymbol{Q}}_{\delta}$ are all on the right-half of the complex plane. The solution to $\underline{\boldsymbol{L}}_{\delta}(u)$ is first given in the next Proposition.

Proposition 1 Let

$$
\tilde{\boldsymbol{M}}_{\delta}(u)=\int_{0}^{u} 2 \tilde{\boldsymbol{\Delta}}_{\sigma^{2}}^{-1} e^{-\left(\tilde{\boldsymbol{Q}}_{\delta}+2 \tilde{\boldsymbol{\Delta}}_{c} \tilde{\boldsymbol{\Delta}}_{\sigma^{2}}^{-1}\right)(u-x)} e^{\tilde{\boldsymbol{Q}}_{\delta} x} d x
$$

and

$$
\tilde{\boldsymbol{g}}_{\delta}(x)=\int_{0}^{x} 2 \tilde{\boldsymbol{\Delta}}_{\sigma^{2}}^{-1} e^{-\left(\tilde{\boldsymbol{Q}}_{\delta}+2 \tilde{\boldsymbol{\Delta}}_{c} \tilde{\boldsymbol{\Delta}}_{\sigma^{2}}^{-1}\right)(x-y)} \mathcal{T}_{\tilde{\boldsymbol{Q}}_{\delta}}\left(\tilde{\boldsymbol{D}}_{1} \circ \tilde{\boldsymbol{f}}(y)\right) d y
$$

Then we have

$$
\underline{\boldsymbol{L}}_{\delta}(u)=\tilde{\boldsymbol{M}}_{\delta}(u)+\int_{0}^{u} \tilde{\boldsymbol{S}}_{\delta}(x) \tilde{\boldsymbol{M}}_{\delta}(u-x) d x, \quad u \geq 0
$$

where

$$
\tilde{\boldsymbol{S}}_{\delta}(x)=\sum_{i=1}^{\infty} \tilde{\boldsymbol{g}}_{\delta}^{* i}(x)
$$

Here the $i$-fold convolution is defined recursively as $\tilde{\boldsymbol{g}}_{\delta}^{* i}(x)=\int_{0}^{x} \tilde{\boldsymbol{g}}_{\delta}^{*(i-1)}(x-y) \tilde{\boldsymbol{g}}_{\delta}(y) d y$ for $i=2,3, \ldots$, with the starting point $\tilde{\boldsymbol{g}}_{\delta}^{* 1}(x)=\tilde{\boldsymbol{g}}_{\delta}(x)$. 
Proof. Because the left-hand side of (3.15) represents a zero matrix by definition, by subtraction we obtain

$$
\begin{aligned}
& \frac{1}{2} s^{2} \tilde{\boldsymbol{\Delta}}_{\sigma^{2}}+s \tilde{\boldsymbol{\Delta}}_{c}-\delta \boldsymbol{E}_{m n}+\tilde{\boldsymbol{D}}_{0}+\tilde{\boldsymbol{D}}_{1} \circ \tilde{\tilde{\boldsymbol{f}}}(s) \\
= & \frac{1}{2}\left(\left(s \boldsymbol{E}_{m n}\right)^{2}-\tilde{\boldsymbol{Q}}_{\delta}^{2}\right) \tilde{\boldsymbol{\Delta}}_{\sigma^{2}}+\left(s \boldsymbol{E}_{m n}-\tilde{\boldsymbol{Q}}_{\delta}\right) \tilde{\boldsymbol{\Delta}}_{c}+\int_{0}^{\infty}\left(e^{-s \boldsymbol{E}_{m n} x}-e^{-\tilde{\boldsymbol{Q}}_{\delta} x}\right)\left(\tilde{\boldsymbol{D}}_{1} \circ \tilde{\boldsymbol{f}}(x)\right) d x \\
= & \left(s \boldsymbol{E}_{m n}-\tilde{\boldsymbol{Q}}_{\delta}\right)\left(\frac{1}{2}\left(s \boldsymbol{E}_{m n}+\tilde{\boldsymbol{Q}}_{\delta}\right) \tilde{\boldsymbol{\Delta}}_{\sigma^{2}}+\tilde{\boldsymbol{\Delta}}_{c}-\mathcal{T}_{s \boldsymbol{E}_{m n}} \mathcal{T}_{\tilde{\boldsymbol{Q}}_{\delta}}\left(\tilde{\boldsymbol{D}}_{1} \circ \tilde{\boldsymbol{f}}(0)\right)\right),
\end{aligned}
$$

where the second step follows from the commutative property (2.2) of Dickson-Hipp operators. Note from (3.13) that the matrix inverse of the above expression is the Laplace transform of $\underline{\boldsymbol{L}}_{\delta}(u)$, namely $\widehat{\underline{\boldsymbol{L}}}_{\delta}(s)$. Hence, by simple manipulations we have that

$$
\begin{aligned}
& \left(\boldsymbol{E}_{m n}-\left(\frac{1}{2}\left(s \boldsymbol{E}_{m n}+\tilde{\boldsymbol{Q}}_{\delta}\right) \tilde{\boldsymbol{\Delta}}_{\sigma^{2}}+\tilde{\boldsymbol{\Delta}}_{c}\right)^{-1} \mathcal{T}_{s \boldsymbol{E}_{m n}} \mathcal{T}_{\tilde{\boldsymbol{Q}}_{\delta}}\left(\tilde{\boldsymbol{D}}_{1} \circ \tilde{\boldsymbol{f}}(0)\right)\right) \underline{\boldsymbol{\boldsymbol { L }}}_{\delta}(s) \\
= & \left(\frac{1}{2}\left(s \boldsymbol{E}_{m n}+\tilde{\boldsymbol{Q}}_{\delta}\right) \tilde{\boldsymbol{\Delta}}_{\sigma^{2}}+\tilde{\boldsymbol{\Delta}}_{c}\right)^{-1}\left(s \boldsymbol{E}_{m n}-\tilde{\boldsymbol{Q}}_{\delta}\right)^{-1} .
\end{aligned}
$$

Because

$$
\left(\frac{1}{2}\left(s \boldsymbol{E}_{m n}+\tilde{\boldsymbol{Q}}_{\delta}\right) \tilde{\boldsymbol{\Delta}}_{\sigma^{2}}+\tilde{\boldsymbol{\Delta}}_{c}\right)^{-1}=2 \tilde{\boldsymbol{\Delta}}_{\sigma^{2}}^{-1}\left(s \boldsymbol{E}_{m n}+\tilde{\boldsymbol{Q}}_{\delta}+2 \tilde{\boldsymbol{\Delta}}_{c} \tilde{\boldsymbol{\Delta}}_{\sigma^{2}}^{-1}\right)^{-1},
$$

inverting the Laplace transforms with respect to $s$ in (3.21) yields the Markov renewal equation

$$
\underline{\boldsymbol{L}}_{\delta}(u)=\int_{0}^{u} \tilde{\boldsymbol{g}}_{\delta}(x) \underline{\boldsymbol{L}}_{\delta}(u-x) d x+\tilde{\boldsymbol{M}}_{\delta}(u), \quad u \geq 0,
$$

where $\tilde{\boldsymbol{M}}_{\delta}(u)$ and $\tilde{\boldsymbol{g}}_{\delta}(x)$ are defined in (3.16) and (3.17), respectively. The matrix $\int_{0}^{\infty} \tilde{\boldsymbol{g}}_{\delta}(x) d x$ is known to be strictly substochastic (see Feng and Shimizu (2014, Appendix D)), and therefore (3.22) can be regarded as a matrix version of defective renewal equation. By Markov renewal theory (e.g. Çinlar (1969, Section 3a) or Asmussen (2003, Section VII.4)), the solution of (3.22) is given by (3.18).

We remark that the Gerber-Shiu function $\phi(u ; \infty)$ in the absence of dividends can in principle be obtained from Feng and Shimizu (2014, Theorem 3.1 and Remark 5.1) via some tedious algebra. Nonetheless, the solution to $\phi(u ; \infty)$ is given in the next Proposition to keep this paper self-contained. We provide a direct proof because some of the techniques will be used later on as well.

\section{Proposition 2 Let}

$$
\tilde{\boldsymbol{Z}}_{\delta}(u)=w(0) \tilde{\boldsymbol{\Delta}}_{\sigma^{2}}^{-1} e^{-\left(\tilde{\boldsymbol{Q}}_{\delta}+2 \tilde{\boldsymbol{\Delta}}_{c} \tilde{\boldsymbol{\Delta}}_{\sigma^{2}}^{-1}\right) u} \tilde{\boldsymbol{\Delta}}_{\sigma^{2}} \boldsymbol{e}_{m n}+\int_{0}^{u} 2 \tilde{\boldsymbol{\Delta}}_{\sigma^{2}}^{-1} e^{-\left(\tilde{\boldsymbol{Q}}_{\delta}+2 \tilde{\boldsymbol{\Delta}}_{c} \tilde{\boldsymbol{\Delta}}_{\sigma^{2}}^{-1}\right)(u-x)} \mathcal{T}_{\tilde{\boldsymbol{Q}}_{\delta}} \underline{\boldsymbol{\zeta}}(x) d x .
$$

Then we have

$$
\underline{\phi}(u ; \infty)=\tilde{\boldsymbol{Z}}_{\delta}(u)+\int_{0}^{u} \tilde{\boldsymbol{S}}_{\delta}(x) \tilde{\boldsymbol{Z}}_{\delta}(u-x) d x, \quad u \geq 0,
$$

where $\tilde{\boldsymbol{S}}_{\delta}(x)$ is defined in (3.19).

Proof. Taking Laplace transforms in (3.10) along with the use of $\underline{\phi}(0 ; \infty)=w(0) \boldsymbol{e}_{m n}$ gives

$$
\left(\frac{1}{2} s^{2} \tilde{\boldsymbol{\Delta}}_{\sigma^{2}}+s \tilde{\boldsymbol{\Delta}}_{c}-\delta \boldsymbol{E}_{m n}+\tilde{\boldsymbol{D}}_{0}+\tilde{\boldsymbol{D}}_{1} \circ \widehat{\tilde{\boldsymbol{f}}}(s)\right) \underline{\widehat{\boldsymbol{\phi}}}(s ; \infty)=\frac{1}{2} \tilde{\boldsymbol{\Delta}}_{\sigma^{2}} \underline{\boldsymbol{\phi}^{\prime}}(0 ; \infty)+w(0)\left(\frac{1}{2} s \tilde{\boldsymbol{\Delta}}_{\sigma^{2}}+\tilde{\boldsymbol{\Delta}}_{c}\right) \boldsymbol{e}_{m n}-\widehat{\widehat{\boldsymbol{\zeta}}}(s) .
$$


Note that the term $\phi^{\prime}(0 ; \infty)$ appearing in the above equation is unknown. Following the same arguments as in the proof of Theorem 2 in Zhang et al. (2011), we omit the straightforward algebra and obtain

$$
\boldsymbol{O}=\frac{1}{2} \tilde{\boldsymbol{\Delta}}_{\sigma^{2}} \underline{\phi}^{\prime}(0 ; \infty)+w(0)\left(\frac{1}{2} \tilde{\boldsymbol{Q}}_{\delta} \tilde{\boldsymbol{\Delta}}_{\sigma^{2}}+\tilde{\boldsymbol{\Delta}}_{c}\right) \boldsymbol{e}_{m n}-\int_{0}^{\infty} e^{-\tilde{\boldsymbol{Q}}_{\delta} u} \underline{\boldsymbol{\zeta}}(u) d u .
$$

By subtraction and property (2.2) of Dickson-Hipp operators, the right-hand side of (3.25) can be represented as

$$
\begin{aligned}
& \frac{1}{2} \tilde{\boldsymbol{\Delta}}_{\sigma^{2}} \underline{\phi}^{\prime}(0 ; \infty)+w(0)\left(\frac{1}{2} s \tilde{\boldsymbol{\Delta}}_{\sigma^{2}}+\tilde{\boldsymbol{\Delta}}_{c}\right) \boldsymbol{e}_{m n}-\underline{\boldsymbol{\zeta}}(s) \\
= & \frac{1}{2} w(0)\left(s \boldsymbol{E}_{m n}-\tilde{\boldsymbol{Q}}_{\delta}\right) \tilde{\boldsymbol{\Delta}}_{\sigma^{2}} \boldsymbol{e}_{m n}+\int_{0}^{\infty}\left(e^{-\tilde{\boldsymbol{Q}}_{\delta} u}-e^{-s \boldsymbol{E}_{m n} u}\right) \underline{\boldsymbol{\zeta}}(u) d u \\
= & \left(s \boldsymbol{E}_{m n}-\tilde{\boldsymbol{Q}}_{\delta}\right)\left(\frac{1}{2} w(0) \tilde{\boldsymbol{\Delta}}_{\sigma^{2}} \boldsymbol{e}_{m n}+\mathcal{T}_{s} \boldsymbol{E}_{m n} \mathcal{T}_{\tilde{\boldsymbol{Q}}_{\delta}} \underline{\boldsymbol{\zeta}}(0)\right) .
\end{aligned}
$$

Substitution of (3.20) and (3.27) into (3.25) yields

$$
\begin{aligned}
& \left(\boldsymbol{E}_{m n}-\left(\frac{1}{2}\left(s \boldsymbol{E}_{m n}+\tilde{\boldsymbol{Q}}_{\delta}\right) \tilde{\boldsymbol{\Delta}}_{\sigma^{2}}+\tilde{\boldsymbol{\Delta}}_{c}\right)^{-1} \mathcal{T}_{s \boldsymbol{E}_{m n}} \mathcal{T}_{\tilde{\boldsymbol{Q}}_{\delta}}\left(\tilde{\boldsymbol{D}}_{1} \circ \tilde{\boldsymbol{f}}(0)\right)\right) \underline{\boldsymbol{\phi}}(s ; \infty) \\
= & \left(\frac{1}{2}\left(s \boldsymbol{E}_{m n}+\tilde{\boldsymbol{Q}}_{\delta}\right) \tilde{\boldsymbol{\Delta}}_{\sigma^{2}}+\tilde{\boldsymbol{\Delta}}_{c}\right)^{-1}\left(\frac{1}{2} w(0) \tilde{\boldsymbol{\Delta}}_{\sigma^{2}} \boldsymbol{e}_{m n}+\mathcal{T}_{s \boldsymbol{E}_{m n}} \mathcal{T}_{\tilde{\boldsymbol{Q}}_{\delta}} \underline{\boldsymbol{\zeta}}(0)\right) .
\end{aligned}
$$

Upon Laplace transform inversion, this leads to the (defective) Markov renewal equation

$$
\underline{\phi}(u ; \infty)=\int_{0}^{u} \tilde{\boldsymbol{g}}_{\delta}(x) \underline{\phi}(u-x ; \infty) d x+\tilde{\boldsymbol{Z}}_{\delta}(u), \quad u \geq 0,
$$

with $\tilde{\boldsymbol{Z}}_{\delta}(u)$ defined in (3.23). Then the solution (3.24) follows immediately.

Remark 1 For a Markov additive risk process under the traditional dividend barrier strategy, Cheung and Landriault (2009) provided the representations of the expected discounted dividends, the higher moments of discounted dividends and the Gerber-Shiu function in their equations (11), (22) and (37), respectively. These formulas were expressed in terms of a homogeneous solution (which the authors denoted by $\boldsymbol{v}^{B}(u)$ ) and the barrier-free Gerber-Shiu function. However, general solutions for these two components were not given. While the barrier-free Gerber-Shiu function can be obtained from our Proposition 2 (with $n=1$ ), the quantity $\boldsymbol{v}^{B}(u)$ is related to the results in Proposition 1 (under $n=1$ ) via $\boldsymbol{v}^{B}(u)=(1 / 2) \underline{\boldsymbol{L}}_{\delta}(u) \boldsymbol{\Delta}_{\sigma^{2}}$.

\subsection{The case $u>b$}

In this subsection, we consider the case $u>b$ for (3.4) and (3.5), which will eventually lead to the full solution to $\underline{\phi}(u ; b)=\left(\phi_{1}^{\top}(u ; b), \ldots, \phi_{n}^{\top}(u ; b)\right)^{\top}$ for $u \geq 0$ as in Theorem 1 . When $u>b$, we note that for $k=1,2, \ldots, n-1$ the equation (3.4) involves both $\phi_{k}(u ; b)$ and $\phi_{k+1}(u ; b)$; whereas (3.5) only involves $\phi_{n}(u ; b)$ as the unknown function. Therefore, our solution procedure is to solve (3.4) for $\phi_{k}(u ; b)$ in terms of $\phi_{k+1}(u ; b)$ recursively for $k=1,2, \ldots, n-1$, with the starting point $\phi_{n}(u ; b)$ obtained as the solution of $(3.5)$. 
First, by some straightforward calculations, we obtain (for $\Re(s)>0$ )

$$
\begin{aligned}
& \int_{b}^{\infty} e^{-s \boldsymbol{E}_{m}(u-b)}\left(\frac{1}{2} \boldsymbol{\Delta}_{\sigma^{2}} \frac{d^{2}}{d u^{2}}+\boldsymbol{\Delta}_{c} \frac{d}{d u}-(\delta+\beta) \boldsymbol{E}_{m}+\boldsymbol{D}_{0}\right) \boldsymbol{\phi}_{k}(u ; b) d u \\
= & \left(\frac{1}{2} s^{2} \boldsymbol{\Delta}_{\sigma^{2}}+s \boldsymbol{\Delta}_{c}-(\delta+\beta) \boldsymbol{E}_{m}+\boldsymbol{D}_{0}\right) \mathcal{T}_{s \boldsymbol{E}_{m}} \boldsymbol{\phi}_{k}(b ; b)-\frac{1}{2} \boldsymbol{\Delta}_{\sigma^{2}} \boldsymbol{\phi}_{k}^{\prime}(b ; b)-\left(\frac{1}{2} s \boldsymbol{\Delta}_{\sigma^{2}}+\boldsymbol{\Delta}_{c}\right) \boldsymbol{\phi}_{k}(b ; b) .
\end{aligned}
$$

Moreover, by a change of order of integrations, it can be shown that

$$
\begin{aligned}
& \int_{b}^{\infty} e^{-s \boldsymbol{E}_{m}(u-b)} \int_{0}^{u}\left(\boldsymbol{D}_{1} \circ \boldsymbol{f}(x)\right) \boldsymbol{\phi}_{k}(u-x ; b) d x d u \\
= & \left(\boldsymbol{D}_{1} \circ \widehat{\boldsymbol{f}}(s)\right) \mathcal{T}_{s \boldsymbol{E}_{m}} \boldsymbol{\phi}_{k}(b ; b)+\int_{0}^{b}\left(\mathcal{T}_{s} \boldsymbol{E}_{m}\left(\boldsymbol{D}_{1} \circ \boldsymbol{f}(b-x)\right)\right) \phi_{k}(x ; b) d x .
\end{aligned}
$$

Hence, multiplying both sides of (3.4) by $e^{-s \boldsymbol{E}_{m}(u-b)}$ and performing integration with respect to $u$ from $b$ to $\infty$, we arrive at

$$
\begin{aligned}
& \left(\frac{1}{2} s^{2} \boldsymbol{\Delta}_{\sigma^{2}}+s \boldsymbol{\Delta}_{c}-(\delta+\beta) \boldsymbol{E}_{m}+\boldsymbol{D}_{0}+\boldsymbol{D}_{1} \circ \widehat{\boldsymbol{f}}(s)\right) \mathcal{T}_{s} \boldsymbol{E}_{m} \boldsymbol{\phi}_{k}(b ; b) \\
= & \frac{1}{2} \boldsymbol{\Delta}_{\sigma^{2}} \boldsymbol{\phi}_{k}^{\prime}(b ; b)+\left(\frac{1}{2} s \boldsymbol{\Delta}_{\sigma^{2}}+\boldsymbol{\Delta}_{c}\right) \boldsymbol{\phi}_{k}(b ; b)-\beta \mathcal{T}_{s \boldsymbol{E}_{m}} \boldsymbol{\phi}_{k+1}(b ; b)-\mathcal{T}_{s} \boldsymbol{E}_{m} \boldsymbol{\zeta}(b) \\
& -\int_{0}^{b}\left(\mathcal{T}_{s} \boldsymbol{E}_{m}\left(\boldsymbol{D}_{1} \circ \boldsymbol{f}(b-x)\right)\right) \boldsymbol{\phi}_{k}(x ; b) d x, \quad k=1,2, \ldots, n-1 .
\end{aligned}
$$

With the use of the matrix $\boldsymbol{Q}_{\gamma}$ defined in Section 2 (under $\gamma=\delta+\beta$ ), analogous to (3.20) one has

$$
\begin{aligned}
& \left(\frac{1}{2} s^{2} \boldsymbol{\Delta}_{\sigma^{2}}+s \boldsymbol{\Delta}_{c}-(\delta+\beta) \boldsymbol{E}_{m}+\boldsymbol{D}_{0}+\boldsymbol{D}_{1} \circ \widehat{\boldsymbol{f}}(s)\right) \\
= & \left(s \boldsymbol{E}_{m}-\boldsymbol{Q}_{\delta+\beta}\right)\left(\frac{1}{2}\left(s \boldsymbol{E}_{m}+\boldsymbol{Q}_{\delta+\beta}\right) \boldsymbol{\Delta}_{\sigma^{2}}+\boldsymbol{\Delta}_{c}-\mathcal{T}_{s} \boldsymbol{E}_{m} \mathcal{T}_{\boldsymbol{Q}_{\delta+\beta}}\left(\boldsymbol{D}_{1} \circ \boldsymbol{f}(0)\right)\right) .
\end{aligned}
$$

Similar to (3.26), the matrix $\boldsymbol{Q}_{\delta+\beta}$ can also be used to determine the condition

$$
\begin{aligned}
\boldsymbol{O}= & \frac{1}{2} \boldsymbol{\Delta}_{\sigma^{2}} \boldsymbol{\phi}_{k}^{\prime}(b ; b)+\left(\frac{1}{2} \boldsymbol{Q}_{\delta+\beta} \boldsymbol{\Delta}_{\sigma^{2}}+\boldsymbol{\Delta}_{c}\right) \boldsymbol{\phi}_{k}(b ; b)-\beta \mathcal{T}_{\boldsymbol{Q}_{\delta+\beta}} \boldsymbol{\phi}_{k+1}(b ; b)-\mathcal{T}_{\boldsymbol{Q}_{\delta+\beta}} \boldsymbol{\zeta}(b) \\
& -\int_{0}^{b}\left(\mathcal{T}_{\boldsymbol{Q}_{\delta+\beta}}\left(\boldsymbol{D}_{1} \circ \boldsymbol{f}(b-x)\right)\right) \boldsymbol{\phi}_{k}(x ; b) d x, \quad k=1,2, \ldots, n-1 .
\end{aligned}
$$

Thus, as in (3.27), the right-hand side of (3.28) can be expressed as

$$
\begin{aligned}
\frac{1}{2} \boldsymbol{\Delta}_{\sigma^{2}} \boldsymbol{\phi}_{k}^{\prime}(b ; b)+ & \left(\frac{1}{2} s \boldsymbol{\Delta}_{\sigma^{2}}+\boldsymbol{\Delta}_{c}\right) \boldsymbol{\phi}_{k}(b ; b)-\beta \mathcal{T}_{s} \boldsymbol{E}_{m} \boldsymbol{\phi}_{k+1}(b ; b)-\mathcal{T}_{s} \boldsymbol{E}_{m} \boldsymbol{\zeta}(b) \\
-\int_{0}^{b}\left(\mathcal{T}_{s \boldsymbol{E}_{m}}\left(\boldsymbol{D}_{1} \circ \boldsymbol{f}(b-x)\right)\right) \boldsymbol{\phi}_{k}(x ; b) d x & \\
=\left(s \boldsymbol{E}_{m}-\boldsymbol{Q}_{\delta+\beta}\right)( & \frac{1}{2} \boldsymbol{\Delta}_{\sigma^{2}} \boldsymbol{\phi}_{k}(b ; b)+\beta \mathcal{T}_{s} \boldsymbol{E}_{m} \mathcal{T}_{\boldsymbol{Q}_{\delta+\beta}} \boldsymbol{\phi}_{k+1}(b ; b)+\mathcal{T}_{s \boldsymbol{E}_{m}} \mathcal{T}_{\boldsymbol{Q}_{\delta+\beta}} \boldsymbol{\zeta}(b) \\
& \left.+\int_{0}^{b}\left(\mathcal{T}_{s} \boldsymbol{E}_{m} \mathcal{T}_{\boldsymbol{Q}_{\delta+\beta}}\left(\boldsymbol{D}_{1} \circ \boldsymbol{f}(b-x)\right)\right) \boldsymbol{\phi}_{k}(x ; b) d x\right), \quad k=1,2, \ldots, n-1 .
\end{aligned}
$$


Plugging (3.29) and (3.30) into (3.28) gives

$$
\begin{aligned}
& \left(\boldsymbol{E}_{m}-\left(\frac{1}{2}\left(s \boldsymbol{E}_{m}+\boldsymbol{Q}_{\delta+\beta}\right) \boldsymbol{\Delta}_{\sigma^{2}}+\boldsymbol{\Delta}_{c}\right)^{-1} \mathcal{T}_{s} \boldsymbol{E}_{m} \mathcal{T}_{\boldsymbol{Q}_{\delta+\beta}}\left(\boldsymbol{D}_{1} \circ \boldsymbol{f}(0)\right)\right) \mathcal{T}_{s} \boldsymbol{E}_{m} \boldsymbol{\phi}_{k}(b ; b) \\
= & \left(\frac{1}{2}\left(s \boldsymbol{E}_{m}+\boldsymbol{Q}_{\delta+\beta}\right) \boldsymbol{\Delta}_{\sigma^{2}}+\boldsymbol{\Delta}_{c}\right)^{-1}\left(\frac{1}{2} \boldsymbol{\Delta}_{\sigma^{2}} \boldsymbol{\phi}_{k}(b ; b)+\beta \mathcal{T}_{s} \boldsymbol{E}_{m} \mathcal{T}_{\boldsymbol{Q}_{\delta+\beta}} \boldsymbol{\phi}_{k+1}(b ; b)+\mathcal{T}_{s} \boldsymbol{E}_{m} \mathcal{T}_{\boldsymbol{Q}_{\delta+\beta}} \boldsymbol{\zeta}(b)\right. \\
& \left.+\int_{0}^{b}\left(\mathcal{T}_{s} \boldsymbol{E}_{m} \mathcal{T}_{\boldsymbol{Q}_{\delta+\beta}}\left(\boldsymbol{D}_{1} \circ \boldsymbol{f}(b-x)\right)\right) \boldsymbol{\phi}_{k}(x ; b) d x\right), \quad k=1,2, \ldots, n-1 .
\end{aligned}
$$

Using the fact that the Dickson-Hipp transform $\mathcal{T}_{s} h(b)$ can be regarded as the Laplace transform (with argument $s$ ) of the shifted function $h(b+\cdot)$ (which extends to matrix quantities), one can perform Laplace transform inversion in the above equation. Together with the application of (3.14) and the continuity condition (3.7), this leads to

$\phi_{k}(b+u ; b)=\int_{0}^{u} \boldsymbol{g}_{\delta+\beta}(x) \phi_{k}(b+u-x ; b) d x+\boldsymbol{W}_{\phi, k}(u)+\boldsymbol{R}_{\phi, k}(u)+\sum_{j=1}^{n} \boldsymbol{H}_{k, j}(u) \boldsymbol{k}_{j}, \quad k=1,2, \ldots, n-1 ; u \geq 0$,

where

$$
\begin{gathered}
\boldsymbol{g}_{\delta+\beta}(x)=2 \boldsymbol{\Delta}_{\sigma^{2}}^{-1} \int_{0}^{x} e^{-\left(\boldsymbol{Q}_{\delta+\beta}+2 \boldsymbol{\Delta}_{c} \boldsymbol{\Delta}_{\sigma^{2}}^{-1}\right)(x-y)} \mathcal{T}_{\boldsymbol{Q}_{\delta+\beta}}\left(\boldsymbol{D}_{1} \circ \boldsymbol{f}(y)\right) d y \\
\boldsymbol{W}_{\phi, k}(u)=2 \beta \boldsymbol{\Delta}_{\sigma^{2}}^{-1} \int_{0}^{u} e^{-\left(\boldsymbol{Q}_{\delta+\beta}+2 \boldsymbol{\Delta}_{c} \boldsymbol{\Delta}_{\sigma^{2}}^{-1}\right)(u-x)} \mathcal{T}_{\boldsymbol{Q}_{\delta+\beta}} \boldsymbol{\phi}_{k+1}(b+x ; b) d x \\
\boldsymbol{R}_{\phi, k}(u)=\boldsymbol{\Delta}_{\sigma^{2}}^{-1} e^{-\left(\boldsymbol{Q}_{\delta+\beta}+2 \boldsymbol{\Delta}_{c} \boldsymbol{\Delta}_{\sigma^{2}}^{-1}\right) u} \boldsymbol{\Delta}_{\sigma^{2}} \boldsymbol{\phi}(b ; \infty)+2 \boldsymbol{\Delta}_{\sigma^{2}}^{-1} \int_{0}^{u} e^{-\left(\boldsymbol{Q}_{\delta+\beta}+2 \boldsymbol{\Delta}_{c} \boldsymbol{\Delta}_{\sigma^{2}}^{-1}\right)(u-x)} \mathcal{T}_{\boldsymbol{Q}_{\delta+\beta} \boldsymbol{\zeta}(b+x) d x} \\
+2 \boldsymbol{\Delta}_{\sigma^{2}}^{-1} \int_{0}^{u} e^{-\left(\boldsymbol{Q}_{\delta+\beta}+2 \boldsymbol{\Delta}_{c} \boldsymbol{\Delta}_{\sigma^{2}}^{-1}\right)(u-y)} \int_{0}^{b}\left(\mathcal{T}_{\boldsymbol{Q}_{\delta+\beta}}\left(\boldsymbol{D}_{1} \circ \boldsymbol{f}(b+y-x)\right)\right) \boldsymbol{\phi}(x ; \infty) d x d y
\end{gathered}
$$

and

$$
\begin{aligned}
\boldsymbol{H}_{k, j}(u)= & \boldsymbol{\Delta}_{\sigma^{2}}^{-1} e^{-\left(\boldsymbol{Q}_{\delta+\beta}+2 \boldsymbol{\Delta}_{c} \boldsymbol{\Delta}_{\sigma^{2}}^{-1}\right) u} \boldsymbol{\Delta}_{\sigma^{2}} \boldsymbol{L}_{\delta, k, j}(b) \\
& +2 \boldsymbol{\Delta}_{\sigma^{2}}^{-1} \int_{0}^{u} e^{-\left(\boldsymbol{Q}_{\delta+\beta}+2 \boldsymbol{\Delta}_{c} \boldsymbol{\Delta}_{\sigma^{2}}^{-1}\right)(u-y)} \int_{0}^{b}\left(\mathcal{T}_{\boldsymbol{Q}_{\delta+\beta}}\left(\boldsymbol{D}_{1} \circ \boldsymbol{f}(b+y-x)\right)\right) \boldsymbol{L}_{\delta, k, j}(x) d x d y, \quad j=1,2 \ldots, n .
\end{aligned}
$$

Clearly, (3.32) is a Markov renewal equation satisfied by $\phi_{k}(b+\cdot ; b)$ (and again $\int_{0}^{\infty} \boldsymbol{g}_{\delta+\beta}(x) d x$ is strictly substochastic). Note that the non-homogeneous term depends on $\phi_{k+1}(b+x ; b)$ for $x>0$ via $\boldsymbol{W}_{\phi, k}(u)$. Upon defining the quantity $\boldsymbol{S}_{\delta+\beta}(x)=\sum_{i=1}^{\infty} \boldsymbol{g}_{\delta+\beta}^{* i}(x)$ with the obvious definition of $i$-fold convolution, by renewal theory and some tedious but straightforward calculations, we arrive at

$$
\begin{aligned}
\phi_{k}(b+u ; b)= & \boldsymbol{W}_{\phi, k}(u)+\boldsymbol{R}_{\phi, k}(u)+\sum_{j=1}^{n} \boldsymbol{H}_{k, j}(u) \boldsymbol{k}_{j} \\
& +\int_{0}^{u} \boldsymbol{S}_{\delta+\beta}(u-z)\left(\boldsymbol{W}_{\phi, k}(z)+\boldsymbol{R}_{\phi, k}(z)+\sum_{j=1}^{n} \boldsymbol{H}_{k, j}(z) \boldsymbol{k}_{j}\right) d z \\
= & \int_{0}^{\infty} \boldsymbol{Z}_{\delta, \beta}(u, y) \phi_{k+1}(b+y ; b) d y+\boldsymbol{K}_{\phi, k}(u)+\sum_{j=1}^{n} \boldsymbol{P}_{k, j}(u) \boldsymbol{k}_{j}, \quad k=1,2, \ldots, n-1 ; u \geq 0,
\end{aligned}
$$


where

$$
\begin{gathered}
\boldsymbol{Z}_{\delta, \beta}(u, y)=2 \beta \boldsymbol{\Delta}_{\sigma^{2}}^{-1} \int_{0}^{\min (u, y)} e^{-\left(\boldsymbol{Q}_{\delta+\beta}+2 \boldsymbol{\Delta}_{c} \boldsymbol{\Delta}_{\sigma^{2}}^{-1}\right)(u-x)} e^{-\boldsymbol{Q}_{\delta+\beta}(y-x)} d x \\
+2 \beta \int_{0}^{u} \int_{0}^{\min (z, y)} \boldsymbol{S}_{\delta+\beta}(u-z) \boldsymbol{\Delta}_{\sigma^{2}}^{-1} e^{-\left(\boldsymbol{Q}_{\delta+\beta}+2 \boldsymbol{\Delta}_{c} \boldsymbol{\Delta}_{\sigma^{2}}^{-1}\right)(z-x)} e^{-\boldsymbol{Q}_{\delta+\beta}(y-x)} d x d z,, \\
\boldsymbol{K}_{\phi, k}(u)=\boldsymbol{R}_{\phi, k}(u)+\int_{0}^{u} \boldsymbol{S}_{\delta+\beta}(u-z) \boldsymbol{R}_{\phi, k}(z) d z,
\end{gathered}
$$

and

$$
\boldsymbol{P}_{k, j}(u)=\boldsymbol{H}_{k, j}(u)+\int_{0}^{u} \boldsymbol{S}_{\delta+\beta}(u-z) \boldsymbol{H}_{k, j}(z) d z, \quad j=1,2 \ldots, n .
$$

Note from (3.33) that $\boldsymbol{R}_{\phi, 1} \equiv \boldsymbol{R}_{\phi, 2} \equiv \cdots \equiv \boldsymbol{R}_{\phi, n-1}$ and therefore (3.37) implies $\boldsymbol{K}_{\phi, 1} \equiv \boldsymbol{K}_{\phi, 2} \equiv \cdots \equiv$ $\boldsymbol{K}_{\phi, n-1}$. We adopt the seemingly redundant subscript to ease presentation later on, as we consider the case $k=n$ next.

For $k=n$, multiplying both sides of (3.5) by $e^{-s \boldsymbol{E}_{m}(u-b)}$ and integrating from $b$ to $\infty$ yields

$$
\begin{aligned}
& \left(\frac{1}{2} s^{2} \boldsymbol{\Delta}_{\sigma^{2}}+s \boldsymbol{\Delta}_{c}-(\delta+\beta) \boldsymbol{E}_{m}+\boldsymbol{D}_{0}+\boldsymbol{D}_{1} \circ \widehat{\boldsymbol{f}}(s)\right) \mathcal{T}_{s \boldsymbol{E}_{m}} \boldsymbol{\phi}_{n}(b ; b) \\
= & \frac{1}{2} \boldsymbol{\Delta}_{\sigma^{2}} \boldsymbol{\phi}_{n}^{\prime}(b ; b)+\left(\frac{1}{2} s \boldsymbol{\Delta}_{\sigma^{2}}+\boldsymbol{\Delta}_{c}\right) \boldsymbol{\phi}_{n}(b ; b)-\beta s^{-1} \boldsymbol{\phi}_{1}(b ; b)-\mathcal{T}_{s} \boldsymbol{E}_{m} \boldsymbol{\zeta}(b) \\
& -\int_{0}^{b}\left(\mathcal{T}_{s} \boldsymbol{E}_{m}\left(\boldsymbol{D}_{1} \circ \boldsymbol{f}(b-x)\right)\right) \boldsymbol{\phi}_{n}(x ; b) d x .
\end{aligned}
$$

We now look at the right-hand side of the above equation. Using the same arguments leading to (3.30) and omitting the details, we obtain

$$
\begin{gathered}
\frac{1}{2} \boldsymbol{\Delta}_{\sigma^{2}} \boldsymbol{\phi}_{n}^{\prime}(b ; b)+\left(\frac{1}{2} s \boldsymbol{\Delta}_{\sigma^{2}}+\boldsymbol{\Delta}_{c}\right) \boldsymbol{\phi}_{n}(b ; b)-\beta s^{-1} \boldsymbol{\phi}_{1}(b ; b)-\mathcal{T}_{s} \boldsymbol{E}_{m} \boldsymbol{\zeta}(b) \\
-\int_{0}^{b}\left(\mathcal{T}_{s} \boldsymbol{E}_{m}\left(\boldsymbol{D}_{1} \circ \boldsymbol{f}(b-x)\right)\right) \boldsymbol{\phi}_{n}(x ; b) d x \\
=\left(s \boldsymbol{E}_{m}-\boldsymbol{Q}_{\delta+\beta}\right)\left(\frac{1}{2} \boldsymbol{\Delta}_{\sigma^{2}} \boldsymbol{\phi}_{n}(b ; b)+\beta\left(s \boldsymbol{Q}_{\delta+\beta}\right)^{-1} \boldsymbol{\phi}_{1}(b ; b)+\mathcal{T}_{s} \boldsymbol{E}_{m} \mathcal{T}_{\boldsymbol{Q}_{\delta+\beta}} \boldsymbol{\zeta}(b)\right. \\
\left.+\int_{0}^{b}\left(\mathcal{T}_{s} \boldsymbol{E}_{m} \mathcal{T}_{\boldsymbol{Q}_{\delta+\beta}}\left(\boldsymbol{D}_{1} \circ \boldsymbol{f}(b-x)\right)\right) \boldsymbol{\phi}_{n}(x ; b) d x\right) .
\end{gathered}
$$

This helps us convert (3.39) into

$$
\begin{aligned}
& \left(\boldsymbol{E}_{m}-\left(\frac{1}{2}\left(s \boldsymbol{E}_{m}+\boldsymbol{Q}_{\delta+\beta}\right) \boldsymbol{\Delta}_{\sigma^{2}}+\boldsymbol{\Delta}_{c}\right)^{-1} \mathcal{T}_{s} \boldsymbol{E}_{m} \mathcal{T}_{\boldsymbol{Q}_{\delta+\beta}}\left(\boldsymbol{D}_{1} \circ \boldsymbol{f}(0)\right)\right) \mathcal{T}_{s} \boldsymbol{E}_{m} \boldsymbol{\phi}_{n}(b ; b) \\
= & \left(\frac{1}{2}\left(s \boldsymbol{E}_{m}+\boldsymbol{Q}_{\delta+\beta}\right) \boldsymbol{\Delta}_{\sigma^{2}}+\boldsymbol{\Delta}_{c}\right)^{-1}\left(\frac{1}{2} \boldsymbol{\Delta}_{\sigma^{2}} \boldsymbol{\phi}_{n}(b ; b)+\beta\left(s \boldsymbol{Q}_{\delta+\beta}\right)^{-1} \boldsymbol{\phi}_{1}(b ; b)+\mathcal{T}_{s} \boldsymbol{E}_{m} \mathcal{T}_{\boldsymbol{Q}_{\delta+\beta}} \boldsymbol{\zeta}(b)\right. \\
& \left.+\int_{0}^{b}\left(\mathcal{T}_{s} \boldsymbol{E}_{m} \mathcal{T}_{\boldsymbol{Q}_{\delta+\beta}}\left(\boldsymbol{D}_{1} \circ \boldsymbol{f}(b-x)\right)\right) \boldsymbol{\phi}_{n}(x ; b) d x\right) .
\end{aligned}
$$

Similar to (3.32), inversion of Laplace transforms yields the defective Markov renewal equation

$$
\phi_{n}(b+u ; b)=\int_{0}^{u} \boldsymbol{g}_{\delta+\beta}(x) \phi_{n}(b+u-x ; b) d x+\boldsymbol{R}_{\phi, n}(u)+\sum_{j=1}^{n} \boldsymbol{H}_{n, j}(u) \boldsymbol{k}_{j}, \quad u \geq 0
$$


where

$$
\begin{aligned}
\boldsymbol{R}_{\phi, n}(u)= & \boldsymbol{\Delta}_{\sigma^{2}}^{-1} e^{-\left(\boldsymbol{Q}_{\delta+\beta}+2 \boldsymbol{\Delta}_{c} \boldsymbol{\Delta}_{\sigma^{2}}^{-1}\right) u} \boldsymbol{\Delta}_{\sigma^{2}} \boldsymbol{\phi}(b ; \infty)+2 \beta \boldsymbol{\Delta}_{\sigma^{2}}^{-1} \int_{0}^{u} e^{-\left(\boldsymbol{Q}_{\delta+\beta}+2 \boldsymbol{\Delta}_{c} \boldsymbol{\Delta}_{\sigma^{2}}^{-1}\right) x} \boldsymbol{Q}_{\delta+\beta}^{-1} \boldsymbol{\phi}(b ; \infty) d x \\
& +2 \boldsymbol{\Delta}_{\sigma^{2}}^{-1} \int_{0}^{u} e^{-\left(\boldsymbol{Q}_{\delta+\beta}+2 \boldsymbol{\Delta}_{c} \boldsymbol{\Delta}_{\sigma^{2}}^{-1}\right)(u-x)} \mathcal{T}_{\boldsymbol{Q}_{\delta+\beta}} \boldsymbol{\zeta}(b+x) d x \\
& +2 \boldsymbol{\Delta}_{\sigma^{2}}^{-1} \int_{0}^{u} e^{-\left(\boldsymbol{Q}_{\delta+\beta}+2 \boldsymbol{\Delta}_{c} \boldsymbol{\Delta}_{\sigma^{2}}^{-1}\right)(u-y)} \int_{0}^{b}\left(\mathcal{T}_{\boldsymbol{Q}_{\delta+\beta}}\left(\boldsymbol{D}_{1} \circ \boldsymbol{f}(b+y-x)\right)\right) \boldsymbol{\phi}(x ; \infty) d x d y
\end{aligned}
$$

and

$$
\begin{aligned}
\boldsymbol{H}_{n, j}(u)= & \boldsymbol{\Delta}_{\sigma^{2}}^{-1} e^{-\left(\boldsymbol{Q}_{\delta+\beta}+2 \boldsymbol{\Delta}_{c} \boldsymbol{\Delta}_{\sigma^{2}}^{-1}\right) u} \boldsymbol{\Delta}_{\sigma^{2}} \boldsymbol{L}_{\delta, n, j}(b)+2 \beta \boldsymbol{\Delta}_{\sigma^{2}}^{-1} \int_{0}^{u} e^{-\left(\boldsymbol{Q}_{\delta+\beta}+2 \boldsymbol{\Delta}_{c} \boldsymbol{\Delta}_{\sigma^{2}}^{-1}\right) x} \boldsymbol{Q}_{\delta+\beta}^{-1} \boldsymbol{L}_{\delta, 1, j}(b) d x \\
& +2 \boldsymbol{\Delta}_{\sigma^{2}}^{-1} \int_{0}^{u} e^{-\left(\boldsymbol{Q}_{\delta+\beta}+2 \boldsymbol{\Delta}_{c} \boldsymbol{\Delta}_{\sigma^{2}}^{-1}\right)(u-y)} \int_{0}^{b}\left(\mathcal{T}_{\boldsymbol{Q}_{\delta+\beta}}\left(\boldsymbol{D}_{1} \circ \boldsymbol{f}(b+y-x)\right)\right) \boldsymbol{L}_{\delta, n, j}(x) d x d y, \quad j=1,2 \ldots, n .
\end{aligned}
$$

As a result, the application of Markov renewal theory gives

$$
\phi_{n}(b+u ; b)=\boldsymbol{K}_{\phi, n}(u)+\sum_{j=1}^{n} \boldsymbol{P}_{n, j}(u) \boldsymbol{k}_{j}, \quad u \geq 0,
$$

where the definitions (3.37) and (3.38) are now extended to $k=n$.

Note that (3.35) for $k=1,2, \ldots, n-1$ forms an iterative system with the starting value given by (3.44). By some straightforward algebra, one can put the iteration in nicer form as

$$
\phi_{n-k+1}(b+u ; b)=\boldsymbol{B}_{\phi, n-k+1}(u)+\sum_{j=1}^{n} \boldsymbol{C}_{n-k+1, j}(u) \boldsymbol{k}_{j}, \quad k=1,2, \ldots, n ; u \geq 0,
$$

where $\boldsymbol{B}_{\phi, n-k+1}(u)$ and $\boldsymbol{C}_{n-k+1, j}(u)$ are evaluated recursively (for increasing $k$ ) as

$$
\left\{\begin{array}{l}
\boldsymbol{B}_{\phi, n}(u)=\boldsymbol{K}_{\phi, n}(u) \\
\boldsymbol{B}_{\phi, n-k+1}(u)=\boldsymbol{K}_{\phi, n-k+1}(u)+\int_{0}^{\infty} \boldsymbol{Z}_{\delta, \beta}(u, y) \boldsymbol{B}_{\phi, n-k+2}(y) d y, \quad k=2,3, \ldots, n
\end{array}\right.
$$

and for each fixed $j=1,2, \ldots, n$,

$$
\left\{\begin{array}{l}
\boldsymbol{C}_{n, j}(u)=\boldsymbol{P}_{n, j}(u) . \\
\boldsymbol{C}_{n-k+1, j}(u)=\boldsymbol{P}_{n-k+1, j}(u)+\int_{0}^{\infty} \boldsymbol{Z}_{\delta, \beta}(u, y) \boldsymbol{C}_{n-k+2, j}(y) d y, \quad k=2,3, \ldots, n .
\end{array}\right.
$$

Furthermore, setting $\underline{\boldsymbol{B}}_{\phi}(u)=\left(\boldsymbol{B}_{\phi, 1}^{\top}(u), \ldots, \boldsymbol{B}_{\phi, n}^{\top}(u)\right)^{\top}$ and

$$
\underline{\boldsymbol{C}}(u)=\left(\begin{array}{ccc}
\boldsymbol{C}_{1,1}(u) & \cdots & \boldsymbol{C}_{1, n}(u) \\
\vdots & \ddots & \vdots \\
\boldsymbol{C}_{n, 1}(u) & \cdots & \boldsymbol{C}_{n, n}(u)
\end{array}\right)
$$

(3.45) can be rewritten as

$$
\underline{\boldsymbol{\phi}}(b+u ; b)=\underline{\boldsymbol{B}}_{\phi}(u)+\underline{\boldsymbol{C}}(u)\left(\boldsymbol{k}_{1}^{\top}, \ldots, \boldsymbol{k}_{n}^{\top}\right)^{\top}, \quad u \geq 0 .
$$


Except for $\left(\boldsymbol{k}_{1}^{\top}, \ldots, \boldsymbol{k}_{n}^{\top}\right)^{\top}$, the above equation gives the exact solution for the Gerber-Shiu function in the upper layer. The unknown vector $\left(\boldsymbol{k}_{1}^{\top}, \ldots, \boldsymbol{k}_{n}^{\top}\right)^{\top}$ can be obtained using the smooth pasting condition (3.8) together with (3.14), giving

$$
\left(\boldsymbol{k}_{1}^{\top}, \ldots, \boldsymbol{k}_{n}^{\top}\right)^{\top}=\left(\underline{\boldsymbol{L}}_{\delta}^{\prime}(b)-\underline{\boldsymbol{C}}^{\prime}(0)\right)^{-1}\left(\underline{\boldsymbol{B}}_{\phi}^{\prime}(0)-\underline{\boldsymbol{\phi}}^{\prime}(b ; \infty)\right) .
$$

The main results of this section are summarized in the following Theorem.

Theorem 1 Suppose that the matrices $\tilde{\boldsymbol{Q}}_{\delta}$ and $\boldsymbol{Q}_{\delta+\beta}$ are diagonalizable. Then

$$
\underline{\phi}(u ; b)=\underline{\phi}(u ; \infty)+\underline{\boldsymbol{L}}_{\delta}(u)\left(\underline{\boldsymbol{L}}_{\delta}^{\prime}(b)-\underline{\boldsymbol{C}}^{\prime}(0)\right)^{-1}\left(\underline{\boldsymbol{B}}_{\phi}^{\prime}(0)-\underline{\phi}^{\prime}(b ; \infty)\right), \quad 0 \leq u \leq b,
$$

and

$$
\underline{\phi}(u ; b)=\underline{\boldsymbol{B}}_{\phi}(u-b)+\underline{\boldsymbol{C}}(u-b)\left(\underline{\boldsymbol{L}}_{\delta}^{\prime}(b)-\underline{\boldsymbol{C}}^{\prime}(0)\right)^{-1}\left(\underline{\boldsymbol{B}}_{\phi}^{\prime}(0)-\underline{\boldsymbol{\phi}}^{\prime}(b ; \infty)\right), \quad u>b .
$$

In particular, the Gerber-Shiu function $\underline{\phi}(u ; b)$ can be computed by the following procedure.

- Step 1: Compute the matrices $\tilde{\boldsymbol{Q}}_{\delta}$ and $\boldsymbol{Q}_{\delta+\beta}$ using one of the methods discussed in Section 2.

- Step 2: Compute $\underline{\boldsymbol{L}}_{\delta}(u)$ and $\underline{\boldsymbol{\phi}}(u ; \infty)$ by Propositions 1 and 2 , respectively.

- Step 3: Compute $\boldsymbol{R}_{\phi, k}(u)$ by (3.33) and (3.42); and $\boldsymbol{H}_{k, j}(u)$ by (3.34) and (3.43).

- Step 4: Compute $\boldsymbol{Z}_{\delta, \beta}(u, y)$ by (3.36); and $\boldsymbol{K}_{\phi, k}(u)$ and $\boldsymbol{P}_{k, j}(u)$ by (3.37) and (3.38), respectively.

- Step 5: Compute $\boldsymbol{B}_{\phi, k}(u)$ and $\boldsymbol{C}_{k, j}(u)$ recursively via (3.46) and (3.47), respectively.

- Step 6: Compute $\underline{\phi}(u ; b)$ via (3.49) and (3.50).

Remark 2 If one is interested in the limit behavior of the Gerber-Shiu function as $u \rightarrow \infty$, it suffices to consider the case $u>b$. Applying the Final Value Theorem for Laplace transforms, we have

$$
\lim _{u \rightarrow \infty} \phi_{k}(u ; b)=\lim _{u \rightarrow \infty} \phi_{k}(b+u ; b)=\lim _{s \rightarrow 0} s \mathcal{T}_{s} \boldsymbol{E}_{m} \phi_{k}(b ; b) .
$$

Hence, application of (3.28) gives the iterative expression

$$
\begin{aligned}
\lim _{s \rightarrow 0} s \mathcal{T}_{s \boldsymbol{E}_{m}} \boldsymbol{\phi}_{k}(b ; b)= & \left(\lim _{s \rightarrow 0}\left(\frac{1}{2} s^{2} \boldsymbol{\Delta}_{\sigma^{2}}+s \boldsymbol{\Delta}_{c}-(\delta+\beta) \boldsymbol{E}_{m}+\boldsymbol{D}_{0}+\boldsymbol{D}_{1} \circ \widehat{\boldsymbol{f}}(s)\right)^{-1}\right) \\
& \times\left(\operatorname { l i m } _ { s \rightarrow 0 } s \left(\frac{1}{2} \boldsymbol{\Delta}_{\sigma^{2}} \boldsymbol{\phi}_{k}^{\prime}(b ; b)+\left(\frac{1}{2} s \boldsymbol{\Delta}_{\sigma^{2}}+\boldsymbol{\Delta}_{c}\right) \boldsymbol{\phi}_{k}(b ; b)-\beta \mathcal{T}_{s} \boldsymbol{E}_{m} \boldsymbol{\phi}_{k+1}(b ; b)-\mathcal{T}_{s \boldsymbol{E}_{m}} \boldsymbol{\zeta}(b)\right.\right. \\
& \left.\left.\quad-\int_{0}^{b}\left(\mathcal{T}_{s \boldsymbol{E}_{m}}\left(\boldsymbol{D}_{1} \circ \boldsymbol{f}(b-x)\right)\right) \boldsymbol{\phi}_{k}(x ; b) d x\right)\right) \\
= & \beta\left((\delta+\beta) \boldsymbol{E}_{m}-\boldsymbol{D}_{0}-\boldsymbol{D}_{1}\right)^{-1} \lim _{s \rightarrow 0} s \mathcal{T}_{s \boldsymbol{E}_{m}} \boldsymbol{\phi}_{k+1}(b ; b), \quad k=1,2, \ldots, n-1 .
\end{aligned}
$$

Similarly, (3.39) leads to the starting point

$$
\lim _{s \rightarrow 0} s \mathcal{T}_{s} \boldsymbol{E}_{m} \boldsymbol{\phi}_{n}(b ; b)=\beta\left((\delta+\beta) \boldsymbol{E}_{m}-\boldsymbol{D}_{0}-\boldsymbol{D}_{1}\right)^{-1} \boldsymbol{\phi}_{1}(b ; b) .
$$

Combining all the above, we arrive at the asymptotic formula, for each $k=1,2, \ldots, n$,

$$
\boldsymbol{\phi}_{k}(u ; b) \sim \beta^{n-k+1}\left((\delta+\beta) \boldsymbol{E}_{m}-\boldsymbol{D}_{0}-\boldsymbol{D}_{1}\right)^{-(n-k+1)} \boldsymbol{\phi}_{1}(b ; b) \quad \text { as } u \rightarrow \infty .
$$


It can be verified that

$$
\int_{0}^{\infty} e^{-\delta t} f_{T}(t) e^{\left(\boldsymbol{D}_{0}+\boldsymbol{D}_{1}\right) t} d t=\beta^{n}\left((\delta+\beta) \boldsymbol{E}_{m}-\boldsymbol{D}_{0}-\boldsymbol{D}_{1}\right)^{-n}
$$

The $(i, j)$ th element of the above expression is $\mathbb{E}\left[e^{-\delta T} \mathbf{1}_{\left\{J_{T}=j\right\}} \mid J_{0}=i\right]$, namely the expected present value of a dollar payable at the first dividend decision time $T_{1}$ if $J$ is in state $j$ at time $T_{1}$, given that $J$ starts in state $i$. Then (3.51) at $k=1$ can be interpreted probabilistically as follows. Suppose $J_{0}=i$. When the initial surplus $U_{0}^{b}=u$ is very large, it is highly likely that the surplus process $U^{b}$ is above $b$ at time $T_{1}$ (before dividends) and ruin has not occurred in the interim, regardless of the initial environmental state $J_{0}=i$. If $J$ is in state $j$ at time $T_{1}$, this first gives rise to the discount factor $\mathbb{E}\left[e^{-\delta T} \mathbf{1}_{\left\{J_{T}=j\right\}} \mid J_{0}=i\right]$ from time $T_{1}$ to time 0 . Then the payment of dividend will cause the surplus to drop to the level $b$, from which the expected discounted penalty onward is $\phi_{1, j}(b ; b)$. Since the state $j$ is arbitrary, summing over $j$ explains (3.51) at $k=1$. Similarly, (3.51) for $k=2,3, \ldots$ can be interpreted by replacing $T_{1}$ with an Erlang $(n-k+1)$ random variable in the above arguments. See Avanzi et al. (2013, Remark 2.3) for related intuitions in the context of a dual risk model.

\section{Expected present value of dividends paid until ruin}

This section is concerned with the full solution to the dividend function $V_{i}(u ; b)$ defined by (1.5). Since the derivations closely resemble those in Section 3 , we mostly present the key steps involved with omission of some algebraic details. As in Section 3, we define $V_{k, i}(u ; b)$ (for $k=1,2, \ldots, n$ ) to be the expected present value of total dividends paid until ruin, given that the time until the first dividend decision time is distributed as Erlang $(n-k+1)$. Clearly, $V_{i}(u ; b)=V_{1, i}(u ; b)$. Let $\boldsymbol{V}_{k}(u ; b)=\left(V_{k, 1}(u ; b), \ldots, V_{k, m}(u ; b)\right)^{\top}$ for $k=1,2, \ldots, n$. Then, applying the same arguments used to obtain (3.4) and (3.5), we arrive at the matrix integro-differential equations

$$
\begin{array}{r}
\boldsymbol{O}=\left(\frac{1}{2} \boldsymbol{\Delta}_{\sigma^{2}} \frac{d^{2}}{d u^{2}}+\boldsymbol{\Delta}_{c} \frac{d}{d u}-(\delta+\beta) \boldsymbol{E}_{m}+\boldsymbol{D}_{0}\right) \boldsymbol{V}_{k}(u ; b)+\int_{0}^{u}\left(\boldsymbol{D}_{1} \circ \boldsymbol{f}(x)\right) \boldsymbol{V}_{k}(u-x ; b) d x+\beta \boldsymbol{V}_{k+1}(u ; b) \\
k=1,2, \ldots, n-1,
\end{array}
$$

and

$$
\begin{aligned}
\boldsymbol{O}= & \left(\frac{1}{2} \boldsymbol{\Delta}_{\sigma^{2}} \frac{d^{2}}{d u^{2}}+\boldsymbol{\Delta}_{c} \frac{d}{d u}-(\delta+\beta) \boldsymbol{E}_{m}+\boldsymbol{D}_{0}\right) \boldsymbol{V}_{n}(u ; b)+\int_{0}^{u}\left(\boldsymbol{D}_{1} \circ \boldsymbol{f}(x)\right) \boldsymbol{V}_{n}(u-x ; b) d x+\beta \boldsymbol{V}_{1}(u ; b) \mathbf{1}_{\{0<u \leq b\}} \\
& +\beta\left((u-b) \boldsymbol{e}_{m}+\boldsymbol{V}_{1}(b ; b)\right) \mathbf{1}_{\{u>b\}} .
\end{aligned}
$$

One has the trivial boundary condition

$$
\boldsymbol{V}_{k}(0 ; b)=\boldsymbol{O}, \quad k=1,2, \ldots, n,
$$

as well as the continuity and the smooth pasting conditions given by

$$
\boldsymbol{V}_{k}(b-; b)=\boldsymbol{V}_{k}(b+; b), \quad k=1,2, \ldots, n,
$$

and

$$
\boldsymbol{V}_{k}^{\prime}(b-; b)=\boldsymbol{V}_{k}^{\prime}(b+; b), \quad k=1,2, \ldots, n .
$$

Setting $\underline{\boldsymbol{V}}(u ; b)=\left(\boldsymbol{V}_{1}^{\top}(u ; b), \ldots, \boldsymbol{V}_{n}^{\top}(u ; b)\right)^{\top},(4.1)$ and (4.2) in the lower layer can be collectively written as

$$
\left(\frac{1}{2} \tilde{\boldsymbol{\Delta}}_{\sigma^{2}} \frac{d^{2}}{d u^{2}}+\tilde{\boldsymbol{\Delta}}_{c} \frac{d}{d u}-\delta \boldsymbol{E}_{m n}+\tilde{\boldsymbol{D}}_{0}\right) \underline{\boldsymbol{V}}(u ; b)+\int_{0}^{u}\left(\tilde{\boldsymbol{D}}_{1} \circ \tilde{\boldsymbol{f}}(x)\right) \underline{\boldsymbol{V}}(u-x ; b) d x=\boldsymbol{O}, \quad 0<u<b .
$$


With the boundary condition (4.3), it follows from Section 3.2 that

$$
\underline{\boldsymbol{V}}(u ; b)=\underline{\boldsymbol{L}}_{\delta}(u)\left(\boldsymbol{y}_{1}^{\top}, \ldots, \boldsymbol{y}_{n}^{\top}\right)^{\top}, \quad 0 \leq u \leq b,
$$

where $\boldsymbol{y}_{1}, \ldots, \boldsymbol{y}_{n}$ are $m$-dimensional column vectors to be determined. For $u>b$, multiplying (4.1) and (4.2) by $e^{-s \boldsymbol{E}_{m}(u-b)}$ and integrating from $b$ to $\infty$, we obtain

$$
\begin{aligned}
&\left(\frac{1}{2} s^{2} \boldsymbol{\Delta}_{\sigma^{2}}+s \boldsymbol{\Delta}_{c}-(\delta+\beta) \boldsymbol{E}_{m}+\boldsymbol{D}_{0}+\boldsymbol{D}_{1} \circ \widehat{\boldsymbol{f}}(s)\right) \mathcal{T}_{s \boldsymbol{E}_{m}} \boldsymbol{V}_{k}(b ; b) \\
&= \frac{1}{2} \boldsymbol{\Delta}_{\sigma^{2}} \boldsymbol{V}_{k}^{\prime}(b ; b)+\left(\frac{1}{2} s \boldsymbol{\Delta}_{\sigma^{2}}+\boldsymbol{\Delta}_{c}\right) \boldsymbol{V}_{k}(b ; b)-\beta \mathcal{T}_{s \boldsymbol{E}_{m}} \boldsymbol{V}_{k+1}(b ; b)-\int_{0}^{b}\left(\mathcal{T}_{s \boldsymbol{E}_{m}}\left(\boldsymbol{D}_{1} \circ \boldsymbol{f}(b-x)\right)\right) \boldsymbol{V}_{k}(x ; b) d x \\
& k=1,2, \ldots, n-1,
\end{aligned}
$$

and

$$
\begin{aligned}
& \left(\frac{1}{2} s^{2} \boldsymbol{\Delta}_{\sigma^{2}}+s \boldsymbol{\Delta}_{c}-(\delta+\beta) \boldsymbol{E}_{m}+\boldsymbol{D}_{0}+\boldsymbol{D}_{1} \circ \widehat{\boldsymbol{f}}(s)\right) \mathcal{T}_{s} \boldsymbol{E}_{m} \boldsymbol{V}_{n}(b ; b) \\
= & \frac{1}{2} \boldsymbol{\Delta}_{\sigma^{2}} \boldsymbol{V}_{n}^{\prime}(b ; b)+\left(\frac{1}{2} s \boldsymbol{\Delta}_{\sigma^{2}}+\boldsymbol{\Delta}_{c}\right) \boldsymbol{V}_{n}(b ; b)-\beta s^{-1} \boldsymbol{V}_{1}(b ; b)-\beta s^{-2} \boldsymbol{e}_{m} \\
& -\int_{0}^{b}\left(\mathcal{T}_{s} \boldsymbol{E}_{m}\left(\boldsymbol{D}_{1} \circ \boldsymbol{f}(b-x)\right)\right) \boldsymbol{V}_{n}(x ; b) d x .
\end{aligned}
$$

Remark 3 As a by-product of (4.7) and (4.8), analogous to (3.51) we can obtain the asymptotic result, for each $k=1,2, \ldots, n$,

$$
\boldsymbol{V}_{k}(u ; b) \sim \beta^{n-k+1}\left((\delta+\beta) \boldsymbol{E}_{m}-\boldsymbol{D}_{0}-\boldsymbol{D}_{1}\right)^{-(n-k+1)} \boldsymbol{e}_{m} u \quad \text { as } u \rightarrow \infty .
$$

The above formula can be interpreted as in Remark 2. See also Avanzi et al. (2013, Remark 3.2).

Following the same steps used to transform (3.28) and (3.39) to (3.31) and (3.40), equations (4.7) and (4.8) respectively become

$$
\begin{aligned}
& \left(\boldsymbol{E}_{m}-\left(\frac{1}{2}\left(s \boldsymbol{E}_{m}+\boldsymbol{Q}_{\delta+\beta}\right) \boldsymbol{\Delta}_{\sigma^{2}}+\boldsymbol{\Delta}_{c}\right)^{-1} \mathcal{T}_{s} \boldsymbol{E}_{m} \mathcal{T}_{\boldsymbol{Q}_{\delta+\beta}}\left(\boldsymbol{D}_{1} \circ \boldsymbol{f}(0)\right)\right) \mathcal{T}_{s} \boldsymbol{E}_{m} \boldsymbol{V}_{k}(b ; b) \\
= & \left(\frac{1}{2}\left(s \boldsymbol{E}_{m}+\boldsymbol{Q}_{\delta+\beta}\right) \boldsymbol{\Delta}_{\sigma^{2}}+\boldsymbol{\Delta}_{c}\right)^{-1}\left(\frac{1}{2} \boldsymbol{\Delta}_{\sigma^{2}} \boldsymbol{V}_{k}(b ; b)+\beta \mathcal{T}_{s} \boldsymbol{E}_{m} \mathcal{T}_{\boldsymbol{Q}_{\delta+\beta}} \boldsymbol{V}_{k+1}(b ; b)\right. \\
& \left.+\int_{0}^{b}\left(\mathcal{T}_{s} \boldsymbol{E}_{m} \mathcal{T}_{\boldsymbol{Q}_{\delta+\beta}}\left(\boldsymbol{D}_{1} \circ \boldsymbol{f}(b-x)\right)\right) \boldsymbol{V}_{k}(x ; b) d x\right), \quad k=1,2, \ldots, n-1,
\end{aligned}
$$

and

$$
\begin{gathered}
\left(\boldsymbol{E}_{m}-\left(\frac{1}{2}\left(s \boldsymbol{E}_{m}+\boldsymbol{Q}_{\delta+\beta}\right) \boldsymbol{\Delta}_{\sigma^{2}}+\boldsymbol{\Delta}_{c}\right)^{-1} \mathcal{T}_{s \boldsymbol{E}_{m}} \mathcal{T}_{\boldsymbol{Q}_{\delta+\beta}}\left(\boldsymbol{D}_{1} \circ \boldsymbol{f}(0)\right)\right) \mathcal{T}_{s} \boldsymbol{E}_{m} \boldsymbol{V}_{n}(b ; b) \\
=\left(\frac{1}{2}\left(s \boldsymbol{E}_{m}+\boldsymbol{Q}_{\delta+\beta}\right) \boldsymbol{\Delta}_{\sigma^{2}}+\boldsymbol{\Delta}_{c}\right)^{-1}\left(\frac{1}{2} \boldsymbol{\Delta}_{\sigma^{2}} \boldsymbol{V}_{n}(b ; b)+\beta\left(s \boldsymbol{Q}_{\delta+\beta}\right)^{-1} \boldsymbol{V}_{1}(b ; b)+\beta s^{-1} \boldsymbol{Q}_{\delta+\beta}^{-2} \boldsymbol{e}_{m}+\beta s^{-2} \boldsymbol{Q}_{\delta+\beta}^{-1} \boldsymbol{e}_{m}\right. \\
\left.+\int_{0}^{b}\left(\mathcal{T}_{s} \boldsymbol{E}_{m} \mathcal{T}_{\boldsymbol{Q}_{\delta+\beta}}\left(\boldsymbol{D}_{1} \circ \boldsymbol{f}(b-x)\right)\right) \boldsymbol{V}_{n}(x ; b) d x\right)
\end{gathered}
$$


Upon inversion of Laplace transforms in (4.10) along with the use of the representation (4.6) and the continuity condition (4.4), we obtain the defective Markov renewal equation

$$
\boldsymbol{V}_{k}(b+u ; b)=\int_{0}^{u} \boldsymbol{g}_{\delta+\beta}(x) \boldsymbol{V}_{k}(b+u-x ; b) d x+\boldsymbol{W}_{V, k}(u)+\sum_{j=1}^{n} \boldsymbol{H}_{k, j}(u) \boldsymbol{y}_{j}, \quad k=1,2, \ldots, n-1 ; u \geq 0
$$

where $\boldsymbol{H}_{k, j}(u)$ is defined in (3.34), and

$$
\boldsymbol{W}_{V, k}(u)=2 \beta \boldsymbol{\Delta}_{\sigma^{2}}^{-1} \int_{0}^{u} e^{-\left(\boldsymbol{Q}_{\delta+\beta}+2 \boldsymbol{\Delta}_{c} \boldsymbol{\Delta}_{\sigma^{2}}^{-1}\right)(u-x)} \mathcal{T}_{\boldsymbol{Q}_{\delta+\beta}} \boldsymbol{V}_{k+1}(b+x ; b) d x .
$$

Similarly, (4.11) leads to

$$
\boldsymbol{V}_{n}(b+u ; b)=\int_{0}^{u} \boldsymbol{g}_{\delta+\beta}(x) \boldsymbol{V}_{n}(b+u-x ; b) d x+\boldsymbol{R}_{V}(u)+\sum_{j=1}^{n} \boldsymbol{H}_{n, j}(u) \boldsymbol{y}_{j}, \quad u \geq 0,
$$

where $\boldsymbol{H}_{n, j}(u)$ is given by (3.43), and

$$
\boldsymbol{R}_{V}(u)=2 \beta \boldsymbol{\Delta}_{\sigma^{2}}^{-1} \int_{0}^{u} e^{-\left(\boldsymbol{Q}_{\delta+\beta}+2 \boldsymbol{\Delta}_{c} \boldsymbol{\Delta}_{\sigma^{2}}^{-1}\right) x} \boldsymbol{Q}_{\delta+\beta}^{-2} \boldsymbol{e}_{m} d x+2 \beta \boldsymbol{\Delta}_{\sigma^{2}}^{-1} \int_{0}^{u} e^{-\left(\boldsymbol{Q}_{\delta+\beta}+2 \boldsymbol{\Delta}_{c} \boldsymbol{\Delta}_{\sigma^{2}}^{-1}\right)(u-x)} x \boldsymbol{Q}_{\delta+\beta}^{-1} \boldsymbol{e}_{m} d x
$$

It is instructive to note that (4.12) and (4.13) are structurally similar to (3.32) and (3.41), respectively. Consequently, in parallel to (3.35) and (3.44), their solutions are

$$
\boldsymbol{V}_{k}(b+u ; b)=\int_{0}^{\infty} \boldsymbol{Z}_{\delta, \beta}(u, y) \boldsymbol{V}_{k+1}(b+y ; b) d y+\sum_{j=1}^{n} \boldsymbol{P}_{k, j}(u) \boldsymbol{y}_{j}, \quad k=1,2, \ldots, n-1 ; u \geq 0,
$$

and

$$
\boldsymbol{V}_{n}(b+u ; b)=\boldsymbol{K}_{V}(u)+\sum_{j=1}^{n} \boldsymbol{P}_{n, j}(u) \boldsymbol{y}_{j} \quad u \geq 0 .
$$

Here the function $\boldsymbol{P}_{k, j}(u)$ has the same definition as in (3.38), and

$$
\boldsymbol{K}_{V}(u)=\boldsymbol{R}_{V}(u)+\int_{0}^{u} \boldsymbol{S}_{\delta+\beta}(u-x) \boldsymbol{R}_{V}(x) d x .
$$

On the grounds of the iterative system that consists of (4.15) and (4.16), we obtain

$$
\boldsymbol{V}_{n-k+1}(b+u ; b)=\boldsymbol{B}_{V, n-k+1}(u)+\sum_{j=1}^{n} \boldsymbol{C}_{n-k+1, j}(u) \boldsymbol{y}_{j}, \quad k=1,2, \ldots, n ; u \geq 0
$$

where $\boldsymbol{C}_{n-k+1, j}(u)$ follows the definition (3.47), and $\boldsymbol{B}_{V, n-k+1}(u)$ can be computed recursively via

$$
\left\{\begin{array}{l}
\boldsymbol{B}_{V, n}(u)=\boldsymbol{K}_{V}(u) . \\
\boldsymbol{B}_{V, n-k+1}(u)=\int_{0}^{\infty} \boldsymbol{Z}_{\delta, \beta}(u, y) \boldsymbol{B}_{V, n-k+2}(y) d y, \quad k=2,3, \ldots, n .
\end{array}\right.
$$

Letting $\underline{\boldsymbol{B}}_{V}(u)=\left(\boldsymbol{B}_{V, 1}^{\top}(u), \ldots, \boldsymbol{B}_{V, n}^{\top}(u)\right)^{\top}$, we can rewrite (4.18) as

$$
\underline{\boldsymbol{V}}(b+u ; b)=\underline{\boldsymbol{B}}_{V}(u)+\underline{\boldsymbol{C}}(u)\left(\boldsymbol{y}_{1}^{\top}, \ldots, \boldsymbol{y}_{n}^{\top}\right)^{\top}, \quad u \geq 0 .
$$

Finally, we further apply (4.6) and the smooth pasting condition (4.5) to determine the unknown vector as

$$
\left(\boldsymbol{y}_{1}^{\top}, \ldots, \boldsymbol{y}_{n}^{\top}\right)^{\top}=\left(\underline{\boldsymbol{L}}_{\delta}^{\prime}(b)-\underline{\boldsymbol{C}}^{\prime}(0)\right)^{-1} \underline{\boldsymbol{B}}_{V}^{\prime}(0) .
$$

We summarize the main results in the following Theorem. 
Theorem 2 Suppose that the matrices $\tilde{\boldsymbol{Q}}_{\delta}$ and $\boldsymbol{Q}_{\delta+\beta}$ are diagonalizable. Then

$$
\underline{\boldsymbol{V}}(u ; b)=\underline{\boldsymbol{L}}_{\delta}(u)\left(\underline{\boldsymbol{L}}_{\delta}^{\prime}(b)-\underline{\boldsymbol{C}}^{\prime}(0)\right)^{-1} \underline{\boldsymbol{B}}_{V}^{\prime}(0), \quad 0 \leq u \leq b,
$$

and

$$
\underline{\boldsymbol{V}}(u ; b)=\underline{\boldsymbol{B}}_{V}(u-b)+\underline{\boldsymbol{C}}(u-b)\left(\underline{\boldsymbol{L}}_{\delta}^{\prime}(b)-\underline{\boldsymbol{C}}^{\prime}(0)\right)^{-1} \underline{\boldsymbol{B}}_{V}^{\prime}(0), \quad u>b .
$$

In particular, the expected discounted dividends paid until ruin $\underline{\boldsymbol{V}}(u ; b)$ can be computed by the following procedure.

- Step 1: Compute the matrices $\tilde{\boldsymbol{Q}}_{\delta}$ and $\boldsymbol{Q}_{\delta+\beta}$ using one of the methods discussed in Section 2.

- Step 2: Compute $\underline{\boldsymbol{L}}_{\delta}(u)$ by Proposition 1.

- Step 3: Compute $\boldsymbol{R}_{V}(u)$ by (4.14); and $\boldsymbol{H}_{k, j}(u)$ by (3.34) and (3.43).

- Step 4: Compute $\boldsymbol{Z}_{\delta, \beta}(u, y)$ by (3.36); and $\boldsymbol{K}_{V}(u)$ and $\boldsymbol{P}_{k, j}(u)$ by (4.17) and (3.38), respectively.

- Step 5: Compute $\boldsymbol{B}_{V, k}(u)$ and $\boldsymbol{C}_{k, j}(u)$ recursively via (4.19) and (3.47), respectively.

- Step 6: Compute $\underline{\boldsymbol{V}}(u ; b)$ via (4.20) and (4.21).

\section{$5 \quad$ Numerical examples}

\subsection{Brownian motion risk model}

In this subsection, we consider the Brownian motion risk model (i.e. $m=1$ and there are no claims at all). Writing $c=c_{1}$ and $\sigma_{1}=\sigma$, the cumulant generating function of the barrier-free process $U^{\infty}$ is given by

$$
\frac{1}{t} \ln \mathbb{E}\left[e^{s U_{t}^{\infty}}\right]=\frac{1}{2} \sigma^{2} s^{2}+c s .
$$

Since there is only one environmental state, we also let $\phi(u ; b)=\phi_{1}(u ; b)$ and $V(u ; b)=V_{1}(u ; b)$. Note that ruin can only be caused by diffusion because there are no claims. Hence, without loss of generality we let $w(0)=1$, and then $\phi(u ; b)$ actually represents the Laplace transform of the time of ruin.

We first assume that the inter-dividend-decision times follow an exponential distribution, i.e. $n=1$. In this simplest case, the results for $\phi(u ; b), V(u ; b)$ and the optimal dividend barrier are very explicit. For the Gerber-Shiu function $\phi(u ; b)$, it follows from $(3.5)$ that

$$
\frac{1}{2} \sigma^{2} \phi^{\prime \prime}(u ; b)+c \phi^{\prime}(u ; b)-(\delta+\beta) \phi(u ; b)+\beta \phi(u ; b) \mathbf{1}_{\{0<u \leq b\}}+\beta \phi(b ; b) \mathbf{1}_{\{u>b\}}=0 .
$$

Due to the simplicity of the problem, instead of using Theorem 1 one can proceed to solve the above piecewise differential equation subject to the conditions

$$
\phi(0 ; b)=1, \quad \phi(b-; b)=\phi(b+; b), \quad \phi^{\prime}(b-; b)=\phi^{\prime}(b+; b),
$$

as well as the fact that $\phi(u ; b) \leq 1$. Following similar notations as in Gerber and Shiu (2004), for $\gamma \geq 0$ we define $s_{\gamma}<0$ and $r_{\gamma} \geq 0$ to be the roots of the quadratic equation (in $\xi$ )

$$
\frac{1}{2} \sigma^{2} \xi^{2}+c \xi-\gamma=0
$$


Omitting the straightforward algebra, we arrive at

$$
\phi(u ; b)=\frac{\left(r_{\delta}-\frac{\delta}{\delta+\beta} s_{\delta+\beta}\right) e^{-s_{\delta}(b-u)}-\left(s_{\delta}-\frac{\delta}{\delta+\beta} s_{\delta+\beta}\right) e^{-r_{\delta}(b-u)}}{\left(r_{\delta}-\frac{\delta}{\delta+\beta} s_{\delta+\beta}\right) e^{-s_{\delta} b}-\left(s_{\delta}-\frac{\delta}{\delta+\beta} s_{\delta+\beta}\right) e^{-r_{\delta} b}}, \quad 0 \leq u \leq b,
$$

and

$$
\phi(u ; b)=\phi(b ; b)\left(\frac{\beta}{\delta+\beta}+\frac{\delta}{\delta+\beta} e^{s_{\delta+\beta}(u-b)}\right), \quad u>b .
$$

For the expected discounted dividends paid until ruin $V(u ; b)$, we obtain from $(4.2)$ that

$$
\frac{1}{2} \sigma^{2} V^{\prime \prime}(u ; b)+c V^{\prime}(u ; b)-(\delta+\beta) V(u ; b)+\beta V(u ; b) \mathbf{1}_{\{0<u \leq b\}}+\beta(u-b+V(b ; b)) \mathbf{1}_{\{u>b\}}=0 .
$$

It can be solved using the conditions

$$
V(0 ; b)=0, \quad V(b-; b)=V(b+; b), \quad V^{\prime}(b-; b)=V^{\prime}(b+; b),
$$

and the fact that $V(u ; b)$ is asymptotically linear in $u$ (see Remark 3 ). This yields

$$
V(u ; b)=\frac{\frac{\beta}{\delta+\beta}\left(1-\frac{c s_{\delta+\beta}}{\delta+\beta}\right)\left(e^{r_{\delta} u}-e^{s_{\delta} u}\right)}{\left(r_{\delta}-\frac{\delta}{\delta+\beta} s_{\delta+\beta}\right) e^{r_{\delta} b}-\left(s_{\delta}-\frac{\delta}{\delta+\beta} s_{\delta+\beta}\right) e^{s_{\delta} b}}, \quad 0 \leq u \leq b,
$$

and

$$
V(u ; b)=V(b ; b)\left(\frac{\beta}{\delta+\beta}+\frac{\delta}{\delta+\beta} e^{s_{\delta+\beta}(u-b)}\right)+\frac{\beta}{\delta+\beta}\left(u-b+\frac{c}{\delta+\beta}\left(1-e^{s_{\delta+\beta}(u-b)}\right)\right), \quad u>b .
$$

Apart from explicit expressions for $\phi(u ; b)$ and $V(u ; b)$, we are also interested in the optimal dividend barrier $b^{*}$ maximizing the dividend function $V(u ; b)$ with respect to $b$. All else being equal, on average a lower (higher) barrier leads to more (less) dividends at early times but less (more) dividends in the long run due to earlier (later) ruin. Hence, choosing $b^{*}$ can somehow be regarded as striking a balance between the timing of dividend payments (because of discounting) and the total (non-discounted) amount of dividends paid. The value of $b^{*}$ can be obtained by solving

$$
\frac{\partial}{\partial b} V(u ; b)=0
$$

From (5.2), one readily obtains

$$
b^{*}=\frac{1}{r_{\delta}-s_{\delta}} \ln \frac{s_{\delta}\left(s_{\delta}-\frac{\delta}{\delta+\beta} s_{\delta+\beta}\right)}{r_{\delta}\left(r_{\delta}-\frac{\delta}{\delta+\beta} s_{\delta+\beta}\right)},
$$

which maximizes $V(u ; b)$ as long as $0 \leq u \leq b^{*}$. It can also be checked that the above $b^{*}$ is also a turning point of the expression (5.3), and therefore it indeed maximizes $V(u ; b)$ for all $u \geq 0$. In parallel to Avanzi et al. (2013, Section 4.3) who considered the optimal barrier in a dual risk model with exponential jumps in the absence of diffusion, we can verify that $V^{\prime}\left(b^{*} ; b^{*}\right)=1$. This leads to

$$
\left.\frac{\partial}{\partial b}[u-b+V(b ; b)]\right|_{b=b^{*}}=0 .
$$

The above equation means that the optimal barrier is still $b^{*}$ even if we declare time 0 to be a dividend decision time. 
Remark 4 Since $s_{\delta+\beta}=\left(-c-\sqrt{c^{2}+2 \sigma^{2}(\delta+\beta)}\right) / \sigma^{2}$, it is clear that $\lim _{\beta \rightarrow \infty} s_{\delta+\beta}=-\infty$ and $\lim _{\beta \rightarrow \infty} s_{\delta+\beta} /(\delta+\beta)=0$. Thus, the limits of (5.1) and (5.2) as $\beta \rightarrow \infty$ are identical to equations (3.7) and (2.11) of Gerber and Shiu (2004), respectively. This is expected because the inter-dividenddecision times tend to zero as $\beta \rightarrow \infty$, and we are back to the traditional barrier strategy in which dividend decisions are made continuously. Moreover, the optimal barrier (5.4) reduces to the one from Gerber and Shiu (2004, equation (6.2)) at the limit. It can also be verified analytically that (5.4) is an increasing concave function of $\beta$ and the details are omitted here.

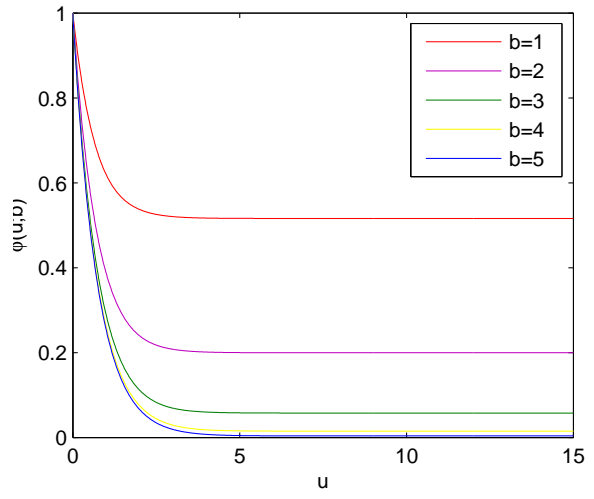

(a)

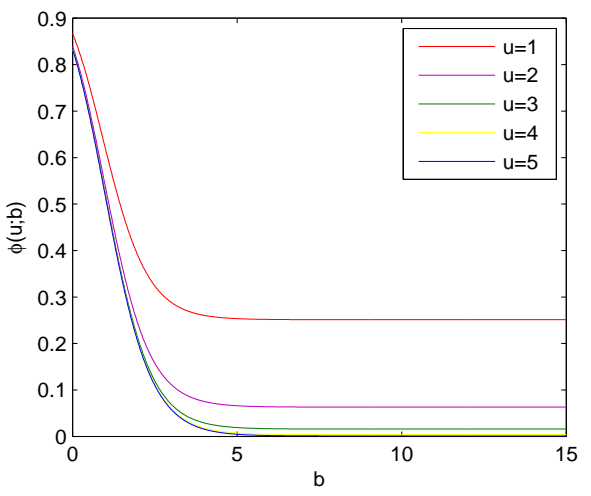

(b)

Figure 1: The Laplace transform of the time of ruin (a) as a function of $u$; and (b) as a function of $b$.

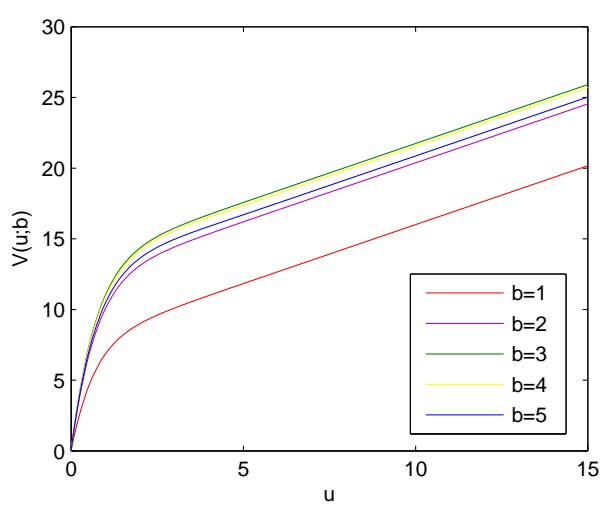

(a)

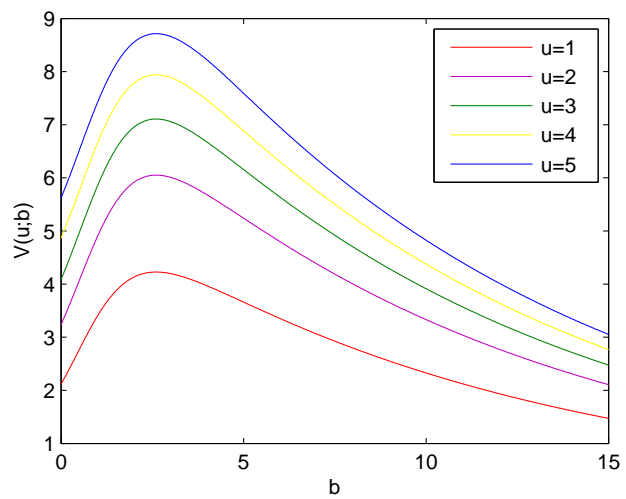

(b)

Figure 2: The expected discounted dividends (a) as a function of $u$; and (b) as a function of $b$.

Based on the explicit formulas derived above, we look at a numerical example by setting $c=0.2$, $\sigma^{2}=0.3, \delta=0.01$ and $\beta=0.05$. Figures $1(\mathrm{a})$ and (b) show the behaviour of the Laplace transform of the time of ruin $\phi(u ; b)$ when either $u$ or $b$ varies. It can be seen that $\phi(u ; b)$ is decreasing in both $u$ and $b$. This is because for larger initial surplus $u$ or larger barrier level $b$, ruin is likely to happen at a later time, and therefore the present value of a dollar payable at ruin is worth less. Note that for each fixed $b$, the curve in Figure 1(a) flattens out as $u$ increases, which can be attributed to the asymptotic formula (3.51). Similarly, the plots of the expected discounted dividends $V(u ; b)$ with respect to $u$ and $b$ are depicted in Figures 2(a) and (b). For each fixed $b$, Figure 2(a) illustrates that $V(u ; b)$ is an increasing function of $u$, and the relationship is almost linear as $u$ increases due to (4.9). However, from Figure 
2(b), for each fixed $u$ the dividend function $V(u ; b)$ is first increasing and then decreasing in $b$, and there is a unique optimal barrier $b^{*}$. In particular, the value of $b^{*}=3.237$ is independent of the initial surplus $u$, which is consistent with our theoretical findings.

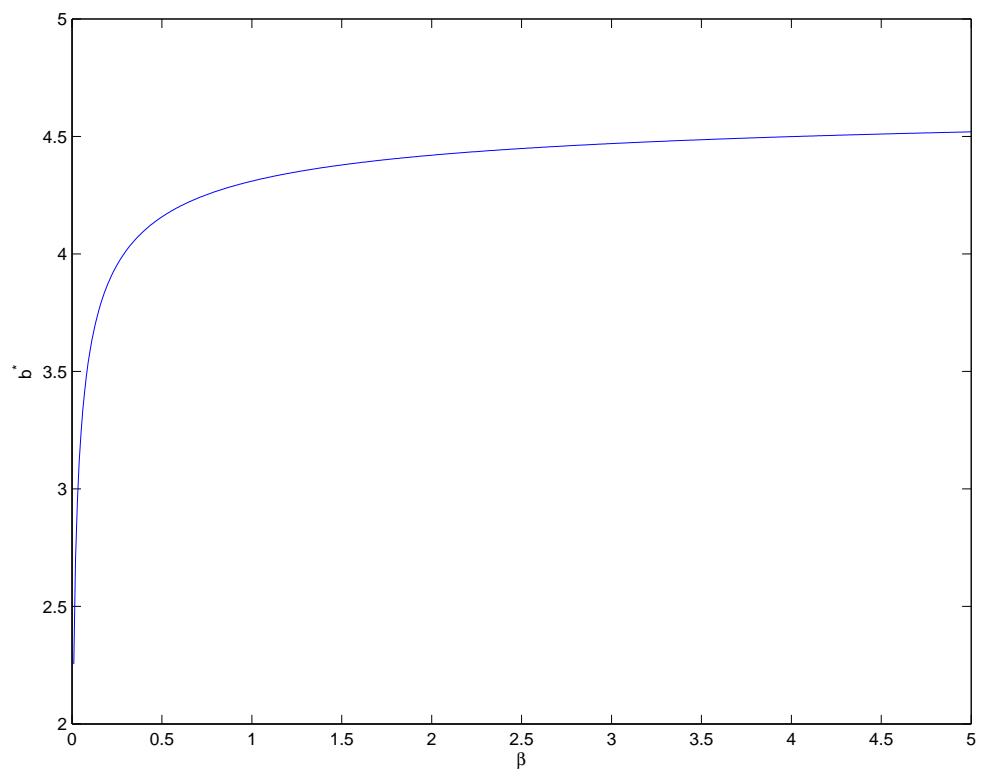

Figure 3: The optimal dividend barrier $b^{*}$ as a function of $\beta=1 / \mathbb{E}[T]$.

Next, we still retain the same parameters except that the value of $\beta$ is varied in order to study its impact on the optimal barrier $b^{*}$. In accordance with Remark 4 , Figure 3 shows that $b^{*}$ is increasing concave in $\beta$, converging to the classical optimal barrier of 4.692 as $\beta \rightarrow \infty$. When $\beta$ increases, the interdividend-decision times are shorter (since $\beta=1 / \mathbb{E}[T]$ ). If the barrier value remains the same, a larger amount of early dividends would be paid at the expense of earlier ruin as dividend decisions are made more frequently, shifting the balance between the timing and the total amount of dividend payments to the former. Thus, the optimal barrier $b^{*}$ should increase to counterbalance the effect of larger $\beta$.

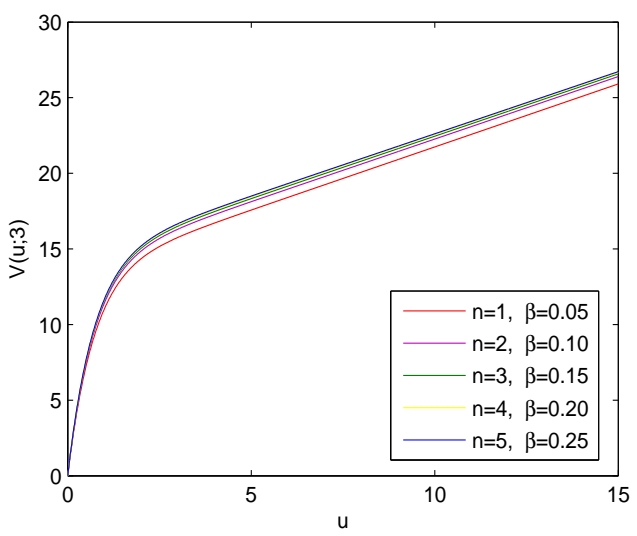

(a)

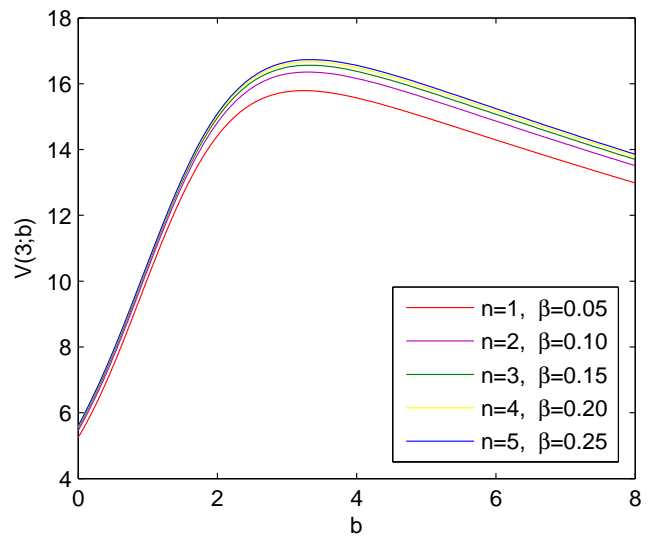

(b)

Figure 4: The expected discounted dividends (a) as a function of $u$ when $b=3$; and (b) as a function of $b$ when $u=3$. 
So far exponential inter-dividend-decision times have been assumed in this subsection. For general $\operatorname{Erlang}(n)$ inter-dividend-decision times with $n>1$, expressions for $\phi(u ; b)$ and $V(u ; b)$ still involve exponential and linear functions in $u$ and $b$ only, but the optimal barrier $b^{*}$ can no longer be represented in explicit form. Instead of directly solving ordinary differential equations, we can use the algorithms provided in Theorems 1 and 2 to get exact values of $\phi(u ; b)$ and $V(u ; b)$. For numerical illustrations, we only look at $V(u ; b)$ since this will give insights to the optimal barrier. We still let $c=0.2, \sigma^{2}=0.3$ and $\delta=0.01$. To see the effect of Erlangization, we increase $n$ from 1 to 5 while fixing $\mathbb{E}[T]=n / \beta=20$ (i.e. increasing $\beta$ ). Figures $4(\mathrm{a})$ and (b) show that $V(u ; 3)$ as a function of $u$ and $V(3 ; b)$ as a function of $b$ are of the same shape as in the case $n=1$ even if we increase $n$. In addition, for fixed values of $u$ and $b$, the dividend function $V(u ; b)$ appears to be increasing and converging as $n$ increases. More importantly, we observe from Figure $4(\mathrm{~b})$ that for each fixed $n$ the optimal barrier $b^{*}$ exists. We have further carried out some numerical checking using different values of initial surplus (which is not reproduced here), and found that for each fixed $n$ the optimal barrier $b^{*}$ is independent of $u$. The values of $b^{*}$ are given by $3.237,3.299,3.300,3.331,3.337$ respectively when $n=1,2,3,4,5$.

\subsection{Bivariate MAP risk model}

This subsection aims at providing further numerical examples for bivariate MAP risk models. First, we consider a bivariate Markov-modulated Brownian risk model (i.e. there are no claims). We set

$$
\boldsymbol{\Delta}_{c}=\left(\begin{array}{cc}
0.1 & 0 \\
0 & 0.25
\end{array}\right), \quad \boldsymbol{\Delta}_{\sigma^{2}}=\left(\begin{array}{cc}
0.1 & 0 \\
0 & 0.2
\end{array}\right), \quad \boldsymbol{D}_{0}=\left(\begin{array}{cc}
-0.06 & 0.06 \\
0.03 & -0.03
\end{array}\right), \quad \boldsymbol{D}_{1}=\boldsymbol{O} .
$$

It is assumed that the inter-dividend-decision times follow the $\operatorname{Erlang}(2)$ distribution with $\beta=0.05$ and the force of interest is $\delta=0.01$.

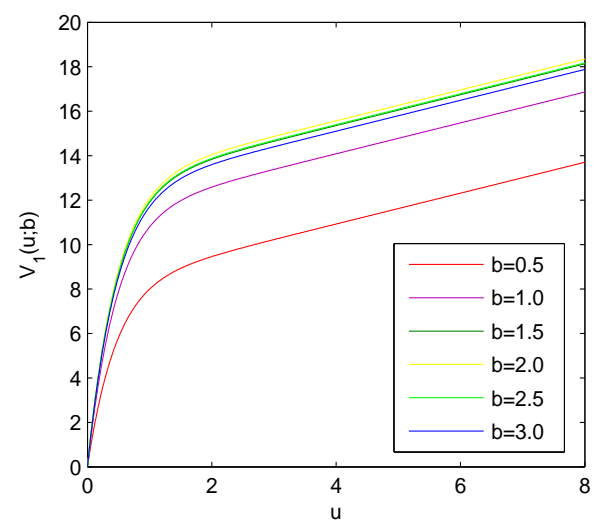

(a)

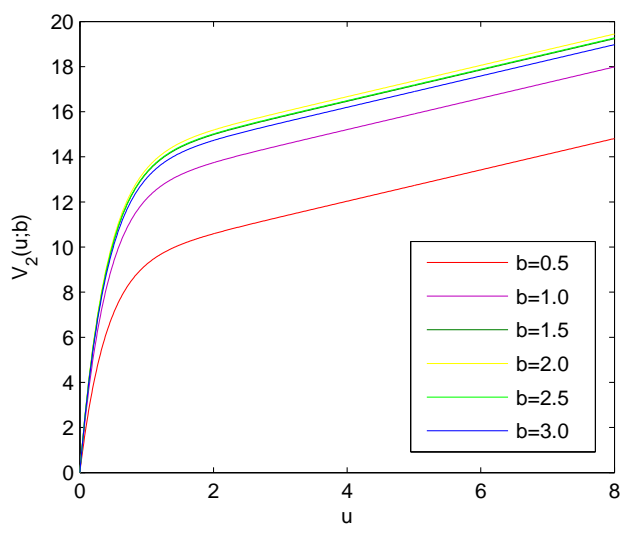

(b)

Figure 5: (a) $V_{1}(u ; b)$ as a function of $u$; and (b) $V_{2}(u ; b)$ as a function of $u$.

From Figure 5, the expected present values of dividends given different initial states, namely $V_{1}(u ; b)$ and $V_{2}(u ; b)$, show similar behaviour as in Figure 2(a), i.e. they both increase in $u$ for each fixed $b$ and then essentially grow linearly as $u$ increases further. For each fixed $u$, Figure 6 shows that $V_{1}(u ; b)$ and $V_{2}(u ; b)$ first increase and then decrease in $b$. Interestingly, using the exact dividend values calculated via Theorem 2, it is found that regardless of the initial surplus level $u$, the optimal dividend barriers that maximize $V_{1}(u ; b)$ and $V_{2}(u ; b)$ coincide and are both given by $b^{*}=1.935$. In other words, the optimal 
dividend barrier $b^{*}$ appears to be independent of the initial surplus and the initial environmental state. The latter also implies that $b^{*}$ is the same for the unconditional process $U^{b}$ under any initial probability row vector $\boldsymbol{\alpha}$ of the Markov chain $J$.

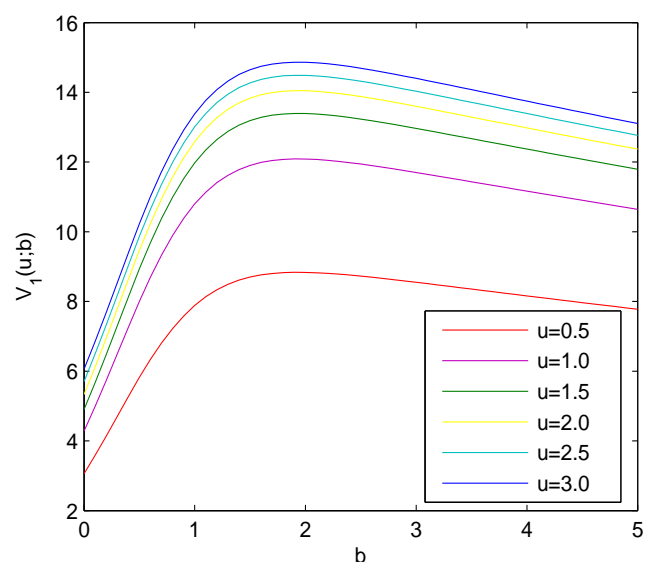

(a)

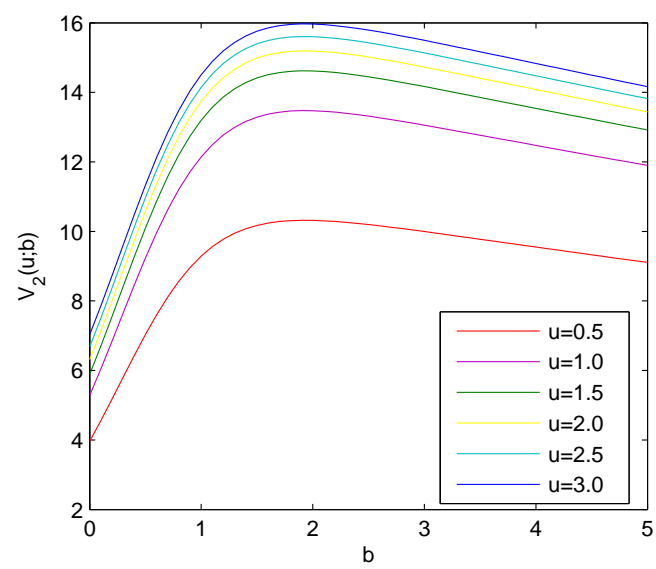

(b)

Figure 6: (a) $V_{1}(u ; b)$ as a function of $b$; and $(b) V_{2}(u ; b)$ as a function of $b$.

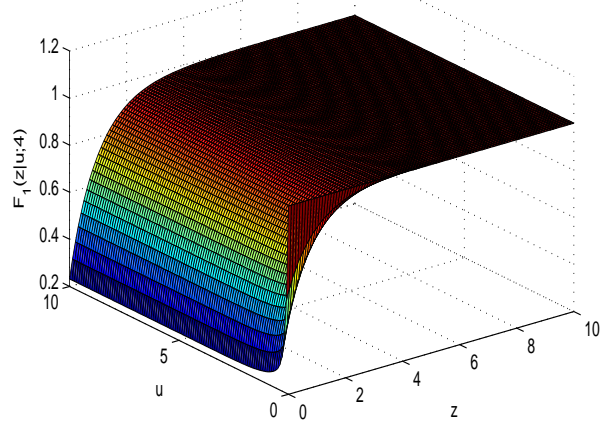

(a)

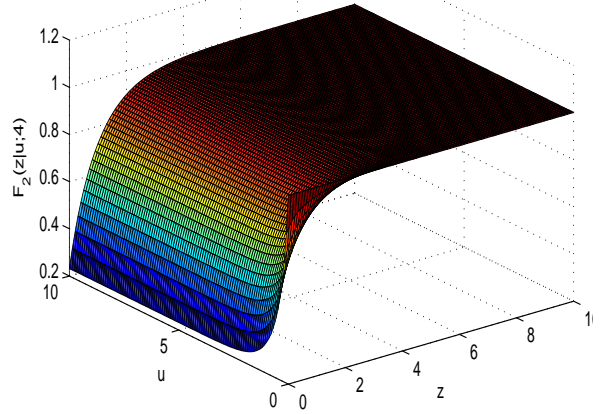

(b)

Figure 7: (a) $F_{1}(z \mid u ; 4)$ as a function of $u$ and $z$; and (b) $F_{2}(z \mid u ; 4)$ as a function of $u$ and $z$.

Next, we look at a perturbed Markov-modulated risk model with two states. Let

$$
\boldsymbol{\Delta}_{c}=\left(\begin{array}{cc}
0.04 & 0 \\
0 & 0.03
\end{array}\right), \quad \boldsymbol{\Delta}_{\sigma^{2}}=\left(\begin{array}{cc}
0.01 & 0 \\
0 & 0.05
\end{array}\right)
$$

and

$$
\boldsymbol{D}_{0}=\left(\begin{array}{cc}
-0.06 & 0.03 \\
0.1 & -0.2
\end{array}\right), \quad \boldsymbol{D}_{1}=\left(\begin{array}{cc}
0.03 & 0 \\
0 & 0.1
\end{array}\right)
$$

The claim severities are assumed to be exponentially distributed such that

$$
f_{11}(x)=e^{-x}, \quad f_{22}(x)=5 e^{-5 x},
$$

so that $\mu_{11}=1$ and $\mu_{22}=0.2$ (and the loading condition (1.2) is satisfied). In addition, the inter-dividenddecision times are assumed to be exponential with $\beta=0.1$. We are interested in the distribution of the 
deficit at ruin, which is denoted by $\boldsymbol{F}(z \mid u ; b)=\left(F_{1}(z \mid u ; b), F_{2}(z \mid u ; b)\right)$. For $i=1,2$, the quantity $F_{i}(z \mid u ; b)$ can be retrieved from $\phi_{i}(u ; b)$ by letting $\delta=0$ and $w(y)=\mathbf{1}_{\{y \leq z\}}$. Figure $7 \operatorname{depicts} \boldsymbol{F}(z \mid u ; b)$ under $b=4$. Note that both $F_{1}(z \mid u ; 4)$ and $F_{2}(z \mid u ; 4)$ have probability masses at $z=0$ because ruin may be caused by oscillation. Furthermore, we observe that $F_{1}(z \mid u ; 4)$ and $F_{2}(z \mid u ; 4)$ tend to 1 as $z$ increases, which is due to the fact that ruin is certain under this Erlangized dividend barrier strategy. Except for small values of $u$ where there is higher chance of early ruin by oscillation, the values of $F_{1}(z \mid u ; 4)$ and $F_{2}(z \mid u ; 4)$ appear to be not very sensitive to change in the initial surplus level.

Finally, we study a bivariate MAP risk process in which $\boldsymbol{\Delta}_{c}$ and $\boldsymbol{\Delta}_{\sigma^{2}}$ are still given by (5.5) and the inter-dividend-decision times are exponential with $\beta=0.1$. However, the generators are now assumed to be

$$
\boldsymbol{D}_{0}=\left(\begin{array}{cc}
-0.06 & 0.03 \\
0.01 & -0.02
\end{array}\right), \quad \boldsymbol{D}_{1}=\left(\begin{array}{cc}
0.02 & 0.01 \\
0 & 0.01
\end{array}\right)
$$

Furthermore, the claim densities are

$$
f_{11}(x)=2 e^{-2 x}, \quad f_{12}(x)=e^{-x}, \quad f_{22}(x)=0.3\left(2 e^{-2 x}\right)+0.7\left(5 e^{-5 x}\right) .
$$

Compared to the previous example, the current specification of $\boldsymbol{D}_{1}$ allows a transition from state 1 to state 2 to be accompanied by a claim (that follows a mixture of two exponentials). We are interested in the Laplace transform of the ruin time given initial state $i$, which can be retrieved from the Gerber-Shiu function $\phi_{i}(u ; b)$ by letting $w \equiv 1$. Under a Laplace transform argument of $\delta=0.01$, the quantities $\phi_{1}(u ; b)$ and $\phi_{2}(u ; b)$ are plotted against the initial surplus $u$ for each fixed $b=1,2,3,4,5$ in Figure 8. Similar behaviour as in Figure 1 is observed, and the same interpretation therein applies.

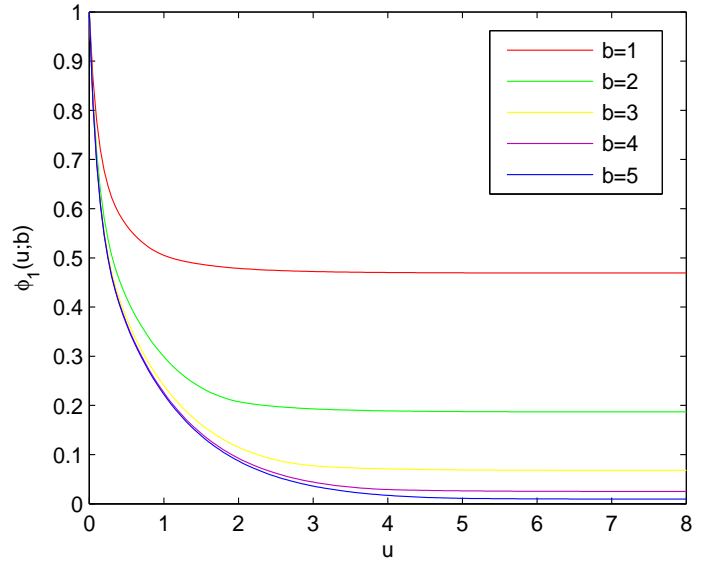

(a)

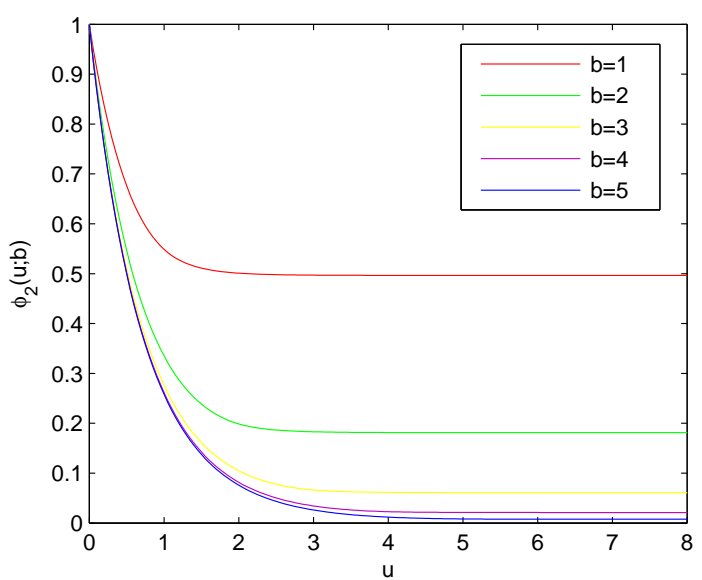

(b)

Figure 8: (a) $\phi_{1}(u ; b)$ as a function of $u$; and $(\mathrm{b}) \phi_{2}(u ; b)$ as a function of $u$.

\section{Acknowledgements}

The authors would like to thank the anonymous referee for helpful comments and suggestions which improved an earlier version of the paper. Zhimin Zhang is supported by the National Natural Science Foundation of China (11101451) and Research Fund for the Doctoral Program of Higher Education of China (20110191120044). Eric Cheung gratefully acknowledges the support from the Research Grants Council of the Hong Kong Special Administrative Region (Project Number: HKU 701212P). 


\section{References}

[1] Ahn, S. And Badescu, A.L. 2007. On the analysis of the Gerber-Shiu discounted penalty function for risk processes with Markovian arrivals. Insurance: Mathematics and Economics 41(2): 234-249.

[2] Ahn, S., Badescu, A.L. and Ramaswami, V. 2007. Time dependent analysis of finite buffer fluid flows and risk models with a dividend barrier. Queueing Systems 55(4): 207-222.

[3] Albrecher, H. And Boxma, O.J. 2005. On the discounted penalty function in a Markovdependent risk model. Insurance: Mathematics and Economics 37(3): 650-672.

[4] Albrecher, H., Cheung, E.C.K. And Thonhauser, S. 2011. Randomized observation periods for the compound Poisson risk model: Dividends. ASTIN Bulletin 41(2): 645-672.

[5] Albrecher, H., Cheung, E.C.K. and Thonhauser, S. 2013. Randomized observation periods for the compound Poisson risk model: The discounted penalty function. Scandinavian Actuarial Journal 2013(6): 424-452.

[6] Asmussen, S. 1989. Risk theory in a Markovian environment. Scandinavian Actuarial Journal 1989(2): 69-100.

[7] Asmussen, S. 2003. Applied Probability and Queues. 2nd Edition. New York: Springer.

[8] Asmussen, S. and Albrecher, H. 2010. Ruin Probabilities. 2nd Edition. New Jersey: World Scientific.

[9] Asmussen, S., Avram, F. And Usabel, M. 2002. Erlangian approximations for finite-horizon ruin probabilities. ASTIN Bulletin 32(2): 267-281.

[10] Avanzi, B., Cheung, E.C.K., Wong, B. And Woo, J.-K. 2013. On a periodic dividend barrier strategy in the dual model with continuous monitoring of solvency. Insurance: Mathematics and Economics 52(1): 98-113.

[11] Badescu, A.L., Breuer, L., Da Silva Soares, A., Latouche, G., Remiche, M.-A. And Stanford, A. 2005. Risk processes analyzed as fluid queues. Scandinavian Actuarial Journal 2005(2): 127-141.

[12] Badescu, A.L., Drekic, S. and Landriault, D. 2007. Analysis of a threshold dividend strategy for a MAP risk model. Scandinavian Actuarial Journal 2007(4): 227-247.

[13] Borodin, A.N. And Salminen, P. 2002. Handbook of Brownian Motion - Facts and Formulae. 2nd Edition. Birkhauser-Verlag: Basel.

[14] Breuer, L. 2008. First passage times for Markov additive processes with positive jumps of phasetype. Journal of Applied Probability 45(3): 779-799.

[15] Cheung, E.C.K. And Feng, R. 2013. A unified analysis of claim costs up to ruin in a Markovian arrival risk model. Insurance: Mathematics and Economics 53(1): 98-109.

[16] Cheung, E.C.K. And Landriault, D. 2009. Perturbed MAP risk models with dividend barrier strategies. Journal of Applied Probability 46(2): 521-541. 
[17] Cheung, E.C.K. And Landriault, D. 2010. A generalized penalty function with the maximum surplus prior to ruin in a MAP risk model. Insurance: Mathematics and Economics 46(1): 127-134.

[18] Çinlar, Erhan. 1969. Markov renewal theory. Advances in Applied Probability 1(2): 123-187.

[19] Dickson, D.C.M. And Hipp, C. 2001. On the time to ruin for Erlang(2) risk processes. Insurance: Mathematics and Economics 29(3): 333-344.

[20] Feng, R. 2009a. On the total operating costs up to default in a renewal risk model. Insurance: Mathematics and Economics 45(2): 305-314.

[21] FEnG, R. 2009b. A matrix operator approach to the analysis of ruin-related quantities in the phasetype renewal risk model. Bulletin of the Swiss Association of Actuaries 2009(1\&2): 71-87.

[22] Feng, R. And Shimizu, Y. 2014. Potential measure of spectrally negative Markov additive process with applications in ruin theory. Preprint.

[23] Gerber, H.U. 1979. An Introduction to Mathematical Risk Theory. Huebner Foundation Monograph 8. Homewood, IL: Richard D. Irwin.

[24] Gerber, H.U. and Landry, B. 1998. On the discounted penalty at ruin in a jump-diffusion and the perpetual put option. Insurance: Mathematics and Economics 22(3): 263-276.

[25] Gerber, H.U. And Shiu, E.S.W. 1998. On the time value of ruin. North American Actuarial Journal 2(1): 48-72.

[26] Gerber, H.U. And Shiu, E.S.W. 2004. Optimal dividends: Analysis with Brownian motion. North American Actuarial Journal 8(1): 1-20.

[27] Ji, L, AND Zhang, C. 2012. Analysis of the multiple roots of the Lundberg fundamental equation in the $\mathrm{PH}(n)$ risk model. Applied Stochastic Models in Business and Industry 28(1): 73-90.

[28] Kyprianou A.E. 2006. Introductory Lectures on Fluctuations of Lévy Processes with Applications. Springer-Verlag: Berlin.

[29] Lin, X.S., Willmot, G.E. And Drekic, S. 2003. The compound Poisson risk model with a constant dividend barrier: analysis of the Gerber-Shiu discounted penalty function. Insurance: Mathematics and Economics 33(3): 551-566.

[30] Lu, Y. AND Tsai, C.C.L. 2007. The expected discounted penalty at ruin for a Markov-modulated risk process perturbed by diffusion. North American Actuarial Journal 11(2): 136-152.

[31] Ramaswami, V., Woolford, D.G. and Stanford, D.A. 2008. The Erlangization method for Markovian fluid flows. Annals of Operations Research 160(1): 215-225.

[32] Salah, Z.B. And Morales, M. 2012. Lévy systems and the time value of ruin for Markov additive processes. European Actuarial Journal 2(2): 289-317.

[33] Stanford, D.A., Avram, F., Badescu, A.L., Breuer, L., Da Silva Soares, A. And Latouche, G. 2005. Phase-type approximations to finite-time ruin probabilities in the SparreAnderson and stationary renewal risk models. ASTIN Bulletin 35(1): 131-144.

[34] Stanford, D.A., Yu, K. and Ren, J. 2011. Erlangian approximation to finite time ruin probabilities in perturbed risk models. Scandinavian Actuarial Journal 2011(1): 38-58. 
[35] Tsai, C.C.L., And Willmot, G.E. 2002. A generalized defective renewal equation for the surplus process perturbed by diffusion. Insurance: Mathematics and Economics 30(1): 51-66.

[36] Zhang, Z. 2014. On a risk model with randomized dividend-decision times. Journal of Industrial and Management Optimization 10(4): 1041-1058.

[37] Zhang, Z., YAng, H. AND YAng, H. 2011. On the absolute ruin in a MAP risk model with debit interest. Advances in Applied Probability 43(1): 77-96.

\section{A Appendix on continuity and smooth pasting}

In this appendix, we demonstrate how to check the continuity conditions (3.7) and (4.4) as well as the smooth pasting conditions (3.8) and (4.5), which have been used to derive full solutions to the Gerber-Shiu function and the expected discounted dividends until ruin. To begin, we need some auxiliary functions. For $i \in \mathcal{E}$ and $u \geq 0$, we define the stopping time $\tau_{i}^{u}=\inf \left\{t>0: u+c_{i} t+\sigma_{i} B_{t}<0\right\}$ and the associated resolvent measure, for $q \geq 0$,

$$
\mathcal{R}_{i}^{(q)}(u, d x)=\int_{0}^{\infty} e^{-q t} \mathbb{P}\left\{u+c_{i} t+\sigma_{i} B_{t} \in d x, t<\tau_{i}^{u}\right\} d t
$$

Further let $\eta_{1 i}^{(q)} \geq 0$ and $\eta_{2 i}^{(q)}<0$ be the roots of the quadratic equation (in $\xi$ )

$$
\frac{1}{2} \sigma_{i}^{2} \xi^{2}+c_{i} \xi-q=0
$$

It follows from Theorem 8.7 and Corollary 8.8 in Kyprianou (2006) that the above resolvent measure admits a density, which is such that $\mathcal{R}_{i}^{(q)}(u, d x)=r_{i}^{(q)}(u, x) d x$ and given by

$$
r_{i}^{(q)}(u, x)=e^{-\eta_{1 i}^{(q)} x} W_{i}^{(q)}(u)-W_{i}^{(q)}(u-x) .
$$

Here $W_{i}^{(q)}$ is a $q$-scale function defined as $W_{i}^{(q)}(x)=0$ for $x<0$ and

$$
W_{i}^{(q)}(x)=\frac{e^{\eta_{1 i}^{(q)} x}-e^{\eta_{2 i}^{(q)} x}}{\frac{\sigma_{i}^{2}}{2}\left(\eta_{1 i}^{(q)}-\eta_{2 i}^{(q)}\right)}, \quad x \geq 0 .
$$

More explicitly, we have

$$
r_{i}^{(q)}(u, x)= \begin{cases}\frac{e^{\eta_{2 i}^{(q)}(u-x)}-e^{\eta_{2 i}^{(q)} u-\eta_{1 i}^{(q)} x}}{\frac{\sigma}{2}^{2}\left(\eta_{1 i}^{(q)}-\eta_{2 i}^{(q)}\right)}, & 0 \leq x \leq u, \\ \frac{e^{\eta_{1 i}^{(q)}(u-x)}-e_{2 i}^{(q)} u-\eta_{1 i}^{(q)} x}{\sigma_{i}^{2}\left(\eta_{1 i}^{(q)}-\eta_{2 i}^{(q)}\right)}, & x>u .\end{cases}
$$

It is also well known that the Laplace transform of $\tau_{i}^{u}$ is (see e.g. Borodin and Salminen (2002, p.295))

$$
\mathcal{H}_{i}^{(q)}(u)=\mathbb{E}\left[e^{-q \tau_{i}^{u}}\right]=e^{\eta_{2 i}^{(q)} u}
$$

The key to proving continuity and smooth pasting is the derivation of appropriate integral equations as follows. Suppose that for the process $U^{b}$, the initial surplus is $u \geq 0$, the initial state is $J_{0}=i \in \mathcal{E}$, and the time until the next dividend decision time is $\operatorname{Erlang}(n-k+1)$ distributed for some $k=1,2, \ldots, n$. 
Let $C_{1}$ be the time until the first phase change of the dividend decision time. Clearly, $C_{1}$ is always exponentially distributed with mean $1 / \beta$. Define $E_{i}$ to be the time until the first event of the bivariate Markov process $(N, J)$ occurs. Then $E_{i}$ is an exponential random variable with mean $-1 / D_{0, i i}$. Three situations need to be distinguished: (1) $\tau^{b}<E_{i} \wedge C_{1}$; (2) $E_{i}<\tau^{b} \wedge C_{1}$; and (3) $C_{1}<\tau^{b} \wedge E_{i}$. Note that under $\mathbb{P}_{u, i}, U^{b}$ is distributed as the process $\left\{u+c_{i} t+\sigma_{i} B_{t}\right\}$ for $0 \leq t<E_{i} \wedge C_{1}$. In addition, $\left\{u+c_{i} t+\sigma_{i} B_{t}\right\}$ and the random variables $E_{i}$ and $C_{1}$ are mutually independent. Therefore, for the Gerber-Shiu function we arrive at

$$
\begin{aligned}
\phi_{k, i}(u ; b)= & w(0) \mathbb{E}\left[e^{\left.-\delta \tau_{i}^{u} \mathbf{1}_{\left\{\tau_{i}^{u}<E_{i}, \tau_{i}^{u}<C_{1}\right\}}\right]}\right. \\
& +\int_{0}^{\infty} e^{-\left(\delta+\beta-D_{0, i i}\right) t} \sum_{j=1, j \neq i}^{m} D_{0, i j} \int_{0}^{\infty} \phi_{k, j}(x ; b) \mathbb{P}\left\{u+c_{i} t+\sigma_{i} B_{t} \in d x, t<\tau_{i}^{u}\right\} d t \\
& +\int_{0}^{\infty} e^{-\left(\delta+\beta-D_{0, i i}\right) t} \sum_{j=1}^{m} D_{1, i j} \int_{0}^{\infty}\left(\gamma_{k, i j}(x ; b)+\omega_{i j}(x)\right) \mathbb{P}\left\{u+c_{i} t+\sigma_{i} B_{t} \in d x, t<\tau_{i}^{u}\right\} d t \\
& +\int_{0}^{\infty} \beta e^{-\left(\delta+\beta-D_{0, i i}\right) t} \int_{0}^{\infty} \phi_{k+1, i}(x ; b) \mathbb{P}\left\{u+c_{i} t+\sigma_{i} B_{t} \in d x, t<\tau_{i}^{u}\right\} d t, \quad k=1,2, \ldots, n-1 .
\end{aligned}
$$

Because

$$
\begin{aligned}
\mathbb{E}\left[e^{-\delta \tau_{i}^{u}} \mathbf{1}_{\left\{\tau_{i}^{u}<E_{i}, \tau_{i}^{u}<C_{1}\right\}}\right] & =\mathbb{E}\left[\mathbb{E}\left[e^{-\delta \tau_{i}^{u}} \mathbf{1}_{\left\{\tau_{i}^{u}<E_{i}, \tau_{i}^{u}<C_{1}\right\}} \mid \tau_{i}^{u}\right]\right]=\mathbb{E}\left[e^{-\delta \tau_{i}^{u}}\left(\int_{\tau_{i}^{u}}^{\infty}\left(-D_{0, i i}\right) e^{D_{0, i i} t} d t\right)\left(\int_{\tau_{i}^{u}}^{\infty} \beta e^{-\beta x} d x\right)\right] \\
& =\mathbb{E}\left[e^{\left.-\left(\delta+\beta-D_{0, i i}\right) \tau_{i}^{u}\right]}=\mathcal{H}^{\left(\delta+\beta-D_{0, i i}\right)}(u),\right.
\end{aligned}
$$

using the resolvent measure we can rewrite (A.2) as

$$
\begin{aligned}
& \phi_{k, i}(u ; b)= w(0) \mathcal{H}^{\left(\delta+\beta-D_{0, i i}\right)}(u)+\sum_{j=1, j \neq i}^{m} D_{0, i j} \int_{0}^{\infty} \phi_{k, j}(x ; b) r_{i}^{\left(\delta+\beta-D_{0, i i}\right)}(u, x) d x \\
&+\sum_{j=1}^{m} D_{1, i j} \int_{0}^{\infty}\left(\gamma_{k, i j}(x ; b)+\omega_{i j}(x)\right) r_{i}^{\left(\delta+\beta-D_{0, i i}\right)}(u, x) d x+\beta \int_{0}^{\infty} \phi_{k+1, i}(x ; b) r_{i}^{\left(\delta+\beta-D_{0, i i}\right)}(u, x) d x \\
& k=1,2, \ldots, n-1 .
\end{aligned}
$$

Similarly, for $k=n$ we have

$$
\begin{aligned}
\phi_{n, i}(u ; b)= & w(0) \mathcal{H}^{\left(\delta+\beta-D_{0, i i}\right)}(u)+\sum_{j=1, j \neq i}^{m} D_{0, i j} \int_{0}^{\infty} \phi_{n, j}(x ; b) r_{i}^{\left(\delta+\beta-D_{0, i i}\right)}(u, x) d x \\
& +\sum_{j=1}^{m} D_{1, i j} \int_{0}^{\infty}\left(\gamma_{n, i j}(x ; b)+\omega_{i j}(x)\right) r_{i}^{\left(\delta+\beta-D_{0, i i}\right)}(u, x) d x+\beta \int_{0}^{b} \phi_{1, i}(x ; b) r_{i}^{\left(\delta+\beta-D_{0, i i}\right)}(u, x) d x \\
& +\beta \int_{b}^{\infty} \phi_{1, i}(b ; b) r_{i}^{\left(\delta+\beta-D_{0, i i}\right)}(u, x) d x
\end{aligned}
$$

Concerning the expected present value of dividends paid until ruin, we have

$$
V_{k, i}(u ; b)=\sum_{j=1, j \neq i}^{m} D_{0, i j} \int_{0}^{\infty} V_{k, j}(x ; b) r_{i}^{\left(\delta+\beta-D_{0, i i}\right)}(u, x) d x
$$




$$
\begin{aligned}
& +\sum_{j=1}^{m} D_{1, i j} \int_{0}^{\infty}\left(\int_{0}^{x} V_{k, j}(x-y ; b) f_{i j}(y) d y\right) r_{i}^{\left(\delta+\beta-D_{0, i i}\right)}(u, x) d x \\
& +\beta \int_{0}^{\infty} V_{k+1, i}(x ; b) r_{i}^{\left(\delta+\beta-D_{0, i i}\right)}(u, x) d x, \quad k=1,2, \ldots, n-1,
\end{aligned}
$$

and

$$
\begin{aligned}
V_{n, i}(u ; b)= & \sum_{j=1, j \neq i}^{m} D_{0, i j} \int_{0}^{\infty} V_{n, j}(x ; b) r_{i}^{\left(\delta+\beta-D_{0, i i}\right)}(u, x) d x \\
& +\sum_{j=1}^{m} D_{1, i j} \int_{0}^{\infty}\left(\int_{0}^{x} V_{n, j}(x-y ; b) f_{i j}(y) d y\right) r_{i}^{\left(\delta+\beta-D_{0, i i}\right)}(u, x) d x \\
& +\beta \int_{0}^{b} V_{1, i}(x ; b) r_{i}^{\left(\delta+\beta-D_{0, i i}\right)}(u, x) d x+\beta \int_{b}^{\infty}\left(x-b+V_{1, i}(b ; b)\right) r_{i}^{\left(\delta+\beta-D_{0, i i}\right)}(u, x) d x .
\end{aligned}
$$

Continuity and smooth pasting can be shown based on (A.3)-(A.6). For illustrative purposes, we only focus on $V_{n, i}(u ; b)$ since the other functions can be checked analogously. Letting

$$
\begin{aligned}
\vartheta_{i}(x)= & \sum_{j=1, j \neq i}^{m} D_{0, i j} V_{n, j}(x ; b)+\sum_{j=1}^{m} D_{1, i j} \int_{0}^{x} V_{n, j}(x-y ; b) f_{i j}(y) d y \\
& +\beta V_{1, i}(x ; b) \mathbf{1}_{\{0<x \leq b\}}+\beta\left(x-b+V_{1, i}(b ; b)\right) \mathbf{1}_{\{x>b\}},
\end{aligned}
$$

(A.6) can be rewritten as

$$
\begin{aligned}
V_{n, i}(u ; b) & =\int_{0}^{\infty} \vartheta_{i}(x) r_{i}^{\left(\delta+\beta-D_{0, i i}\right)}(u, x) d x \\
& =\int_{0}^{u} \vartheta_{i}(x) \frac{e^{\tilde{\eta}_{2 i}(u-x)}-e^{\tilde{\eta}_{2 i} u-\tilde{\eta}_{1 i} x}}{\frac{\sigma_{i}^{2}}{2}\left(\tilde{\eta}_{1 i}-\tilde{\eta}_{2 i}\right)} d x+\int_{u}^{\infty} \vartheta_{i}(x) \frac{e^{\tilde{\eta}_{1 i}(u-x)}-e^{\tilde{\eta}_{2 i} u-\tilde{\eta}_{1 i} x}}{\frac{\sigma_{i}^{2}}{2}\left(\tilde{\eta}_{1 i}-\tilde{\eta}_{2 i}\right)} d x .
\end{aligned}
$$

Here (A.1) has been used in the second equality, and we define $\tilde{\eta}_{1 i}=\eta_{1 i}^{\left(\delta+\beta-D_{0, i i}\right)}$ and $\tilde{\eta}_{2 i}=\eta_{2 i}^{\left(\delta+\beta-D_{0, i i}\right)}$ for convenience. From the above representation, it is clear that $V_{n, i}(0 ; b)=0$ and $V_{n, i}(u ; b)$ is a continuous function in $u$ for all $u \geq 0$. Similarly, one can deduce from (A.5) that $V_{k, i}(u ; b)$ is continuous in $u$ for each $k=1,2, \ldots, n-1$. Consequently, $\vartheta_{i}(x)$ is continuous as well, as evident from (A.7). Hence, taking derivative of (A.8) with respect to $u$ gives

$$
V_{n, i}^{\prime}(u ; b)=\int_{0}^{u} \vartheta_{i}(x) \frac{\tilde{\eta}_{2 i} e^{\tilde{\eta}_{2 i}(u-x)}-\tilde{\eta}_{2 i} e^{\tilde{\eta}_{2 i} u-\tilde{\eta}_{1 i} x}}{\frac{\sigma^{2}}{2}\left(\tilde{\eta}_{1 i}-\tilde{\eta}_{2 i}\right)} d x+\int_{u}^{\infty} \vartheta_{i}(x) \frac{\tilde{\eta}_{1 i} e^{\tilde{\eta}_{1 i}(u-x)}-\tilde{\eta}_{2 i} e^{\tilde{\eta}_{2 i} u-\tilde{\eta}_{1 i} x}}{\frac{\sigma^{2}}{2}\left(\tilde{\eta}_{1 i}-\tilde{\eta}_{2 i}\right)} d x .
$$

Therefore, $V_{n, i}^{\prime}(u ; b)$ is continuous in $u$ for all $u \geq 0$. Indeed, by further differentiating the above equation with respect to $u$ (or by inspecting (4.2) and using the fact that $V_{n, i}(u ; b)$ and $V_{n, i}^{\prime}(u ; b)$ are continuous in $u$ ), one can observe that $V_{n, i}^{\prime \prime}(u ; b)$ is also continuous in $u$ for all $u \geq 0$. However, higher order derivatives are in general not continuous at $u=b$. 